Progress in Flight Physics 5 (2013) 247-284

DOI: $10.1051 /$ eucass/201305247

(C) Owned by the authors, published by EDP Sciences, 2013

\title{
NUMERICAL ANALYSIS OF THREE COMPLEX THREE-DIMENSIONAL SHOCK WAVE / TURBULENT BOUNDARY LAYER INTERACTION FLOWS
}

\author{
A. D. Grosvenor ${ }^{1}$, A. A. Zheltovodov ${ }^{2}$, M. A. Matheson ${ }^{3}$, \\ L. M. Sailer ${ }^{1}$, M. Krzysztopik ${ }^{1}$, and D. P. Gutzwiller ${ }^{4}$ \\ ${ }^{1}$ Ramgen Power Systems, LLC \\ Bellevue WA, USA \\ ${ }^{2}$ Khristianovich Institute of Theoretical and Applied Mechanics \\ Siberian Branch of the Russian Academy of Sciences \\ Novosibirsk, Russia \\ ${ }^{3}$ Oak Ridge National Laboratory \\ Oak Ridge TN, USA \\ ${ }^{4}$ Numeca USA \\ San Francisco CA, USA
}

\begin{abstract}
A set of Reynolds-averaged Navier-Stokes (RANS) computations with turbulence closure provided by the Spalart-Allmaras (SA) model have been carried out for prediction of shock-induced three-dimensional (3D) turbulent separated flows. The experimental data for different aerodynamic test configurations have been used for assessing credibility of the numerical method employed. Particularly, shock wave/turbulent boundary layer interactions (SWTBLI) in the vicinity of an asymmetric sharp double-fin (DF) with different $\left(7^{\circ}\right.$ and $\left.11^{\circ}\right)$ deflection angles mounted on a flat plate and two conically sharp cylindrical bodies at varying interbody distances and nose cone angles $60^{\circ}$ and $40^{\circ}$ mounted over a flat plate at freestream Mach number 4 , as well as a transonic fan stage operating in the near-stall regime are considered. The gas dynamic structure and topology of 3D separated flows, surface flow patterns, and pressure distributions as well as body aerodynamic force prediction are analyzed. A transonic fan stage operating in the near-stall regime and a possibility of applying flow control is investigated. High Performance Computing was employed to make high resolution computations of these flows possible, and advanced $3 \mathrm{D}$ visualization techniques were employed in order to improve understanding of the separating flow phenomena.
\end{abstract}




\section{INTRODUCTION}

Analysis of SWTBLI and development of efficient methods for their modeling are a challenging problem in current aerodynamics. Effective design of modern high-speed air vehicles requires deep understanding of specific physical processes which accompany such interactions occurring in different situations. Examples include high-speed inlets, nozzles, deflected control surfaces, transonic compressors as well as aerodynamic interference of various bodies at supersonic flight. Three-dimensional turbulent separated flows arising under such conditions are especially complex and continue to be studied actively $[1,2]$. Collaborative experimental and theoretical research are crucial to the understanding of their flowfield structure and achieving reliable prediction for various engineering applications.

It is well known that all turbulent separated flows vary in time to a certain degree in fact, and consistency of different relevant numerical modeling techniques are under intensive research today (see, e.g., [1-6]). Two primary modern approaches for simulating unsteady turbulence are developed: (i) Direct Numerical Simulation (DNS); and (ii) Large Eddy Simulation (LES). Some progress in applying such approaches for predicting SWTBLI has been demonstrated in [4-6]. As was shown, existing examples are limited by the Reynolds numbers $\operatorname{Re}_{\delta}=24000-60000$ (and, respectively, $\operatorname{Re}_{\theta}=2400-6000$ ) which are far below that required for the analysis of modern flight configurations and compressors. Here, $\delta$ and $\theta$ represent boundary layer thickness and momentum thickness immediately upstream of interaction region.

Another approach is the numerical solution of RANS equations incorporating semiempirical turbulence models. This approach is based on assumptions related to a steady flow and is used widely for predicting some "mean-flow" SWTBLI characteristics at the higher Reynolds numbers for various practical problems. Although some mean-flow separation properties can be captured reasonably well by RANS techniques, there are limits to what they can be used due to the pronounced intermittent nature or heightened unsteadiness of turbulent separated flows at some interaction regimes, as well as due to limitations of existing turbulence models [1-6].

A compromise approach that has been devised is Detached Eddy Simulation (DES) [7]. This is a hybrid approach where RANS is applied to attached flow and regions of separated flow are intelligently switched to LES. The intelligent switching procedure is still a topic of research, as nonphysical switching can occur, for instance, due to certain types of clustering in the computational grid. The hybrid LES/RANS approach has a perspective for predicting unsteady and 3D effects at higher Reynolds numbers (see, e.g., [8]) as compared to that achieved by LES [9]; however, the computational cost of such calculations remains very high even for predicting flow over simplified test configurations. 


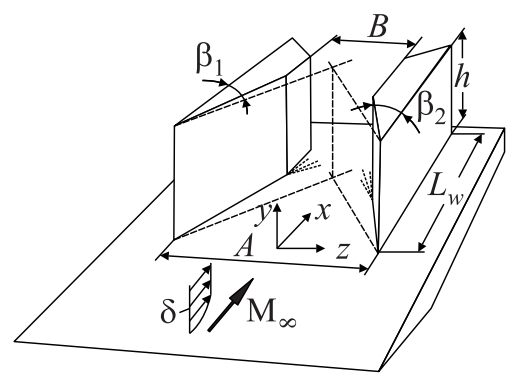

Figure 1 Asymmetric DF test configuration

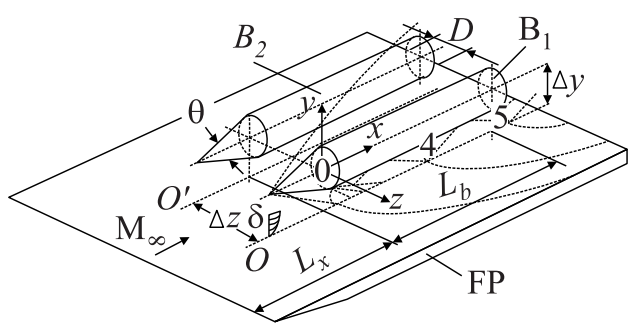

Figure 2 Double-body (DB) test configuration: $\mathrm{B}_{1}$ - first body; $\mathrm{B}_{2}$ - second body; and FP - flat plate

In accordance with analysis of developing different numerical concepts for predicting SWTBLI (see, e.g., $[2,4,6]$ ), it was concluded that RANS models will continue to remain the standard for engineering applications in the near future. This paper presents some 3D SWTBLI examples where RANS Computational Fluid Dynamics (CFD) predictions are compared with experimental data for 3 test cases: (a) asymmetric $\left(\beta_{1} \neq \beta_{2}\right)$ sharp DF configuration (Fig. 1); (b) conically sharp cylindrical bodies mounted over a flat plate (Fig. 2); and (c) a transonic compressor configuration (Fig. 3). Detailed description of the test configurations and the flow conditions are provided below.

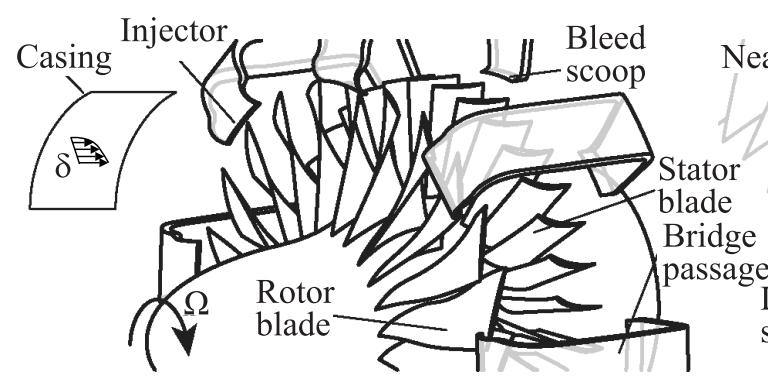

(a)

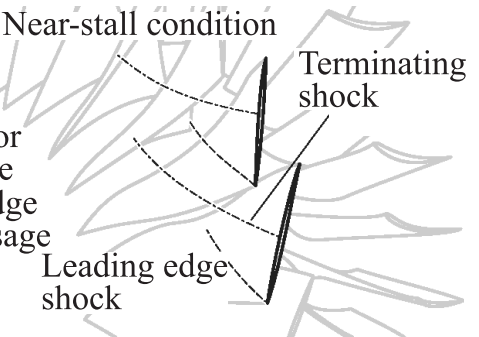

(b)

Figure 3 Transonic fan with tip injection: schematic of Stage 67 configuration $(a)$ and rotor shock system at near-stall operating conditions $(b)$ 


\section{Numerical Models}

The Numeca FINE/Turbo (F/T) compressible Navier-Stokes code was used, employing a cell-centered 2nd order finite volume density based scheme [10] that has been modeled after the 4-stage Runge-Kutta time integration method of Jameson-Schmidt-Turkel (JST) [11]. Geometric multigrid convergence acceleration is employed and $\mathrm{F} / \mathrm{T}$ conducts solutions on structured multiblock grids. The SA one-equation closure [12] was chosen based on a series of earlier relevant studies. Prior supporting discussion of the method and CFD validation of $F / T$ was presented in [13-16].

In all three cases, the grids have been generated for turbulence model sublayer integration, and the near-wall resolution has been set to obtain values $y_{1}^{+} \ll 1$. The grids employed herein have been generated to include much higher resolution than earlier computations presented in [13-16]. In the present cases, a desire to visualize clear 3D shock structures and to plan for future employment of hybrid RANS/LES turbulence modeling approaches drove the grid sizes into the tens and hundreds of millions of cells. High-resolution computations have been accomplished through the application of high performance computing, using a 128 core SGI Altix cluster at Ramgen Power Systems, and the Oak Ridge National Labs Jaguar Cray XT5 supercomputer. Advanced visualization techniques have been employed to provide the detailed views of the 3D flowfields.

\section{ASYMMETRIC DOUBLE-FIN CROSSING SHOCK FLOW}

\subsection{Flow Conditions and Grid}

Considered asymmetric $\left(\beta_{1} \times \beta_{2}=7^{\circ} \times 11^{\circ}\right)$ sharp DF configuration (see Fig. 1) in accordance with experiments [17] at the free-stream incoming Mach number $\mathrm{M}_{\infty}=3.9$ is interesting due to the crossing shock interaction with a turbulent boundary layer on the plate surface which is realized in high-speed inlets. The chamfered fins of length $L_{w}=192 \mathrm{~mm}$ and height $h=100 \mathrm{~mm}$ located $210 \mathrm{~mm}$ downstream of the plate leading edge, separated at the entrance by the distance $A=73.5 \mathrm{~mm}$ with a minimum throat width $B=32 \mathrm{~mm}$. The details of the flow conditions are presented in Table 1 , where $\delta$ and $\delta^{*}$ are the boundary layer and displacement thicknesses, respectively, in the vicinity of the fins leading edges at the adiabatic wall conditions corresponding to free-stream total temperature $T_{t \infty}$.

The computational grid is indicated in Fig. 4. The grid was constructed based on a prior validation study [13], but much higher resolution grid refinement was applied to the passage between the fins presently, and the resultant grid contained 
Table 1 Experimental and computational conditions for DF test case

\begin{tabular}{cccccc}
\hline $\mathrm{M}_{\infty}$ & $\mathrm{Re}_{\delta}$ & $P_{t \infty}, \mathrm{MPa}$ & $T_{t \infty}, \mathrm{K}$ & $\delta, \mathrm{mm}$ & $\delta^{*}, \mathrm{~mm}$ \\
\hline 3.9 & $3.1 \cdot 10^{5}$ & 1.5 & 260 & 3.5 & 1.1 \\
\hline
\end{tabular}
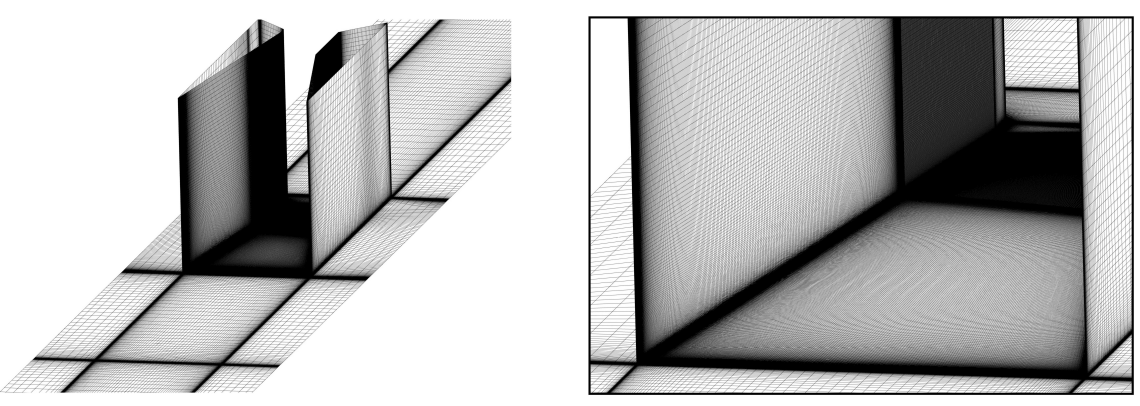

Figure 4 Computational grids

300 million grid cells. Uniform static conditions were set at the inflow boundary, and a turbulent boundary layer was developed upstream of the wedges by a slip wall and flat plate configuration. The outflow boundary was set to a supersonic condition which extrapolates all quantities from the upstream domain. Side and top boundaries were set to slip walls.

\subsection{Results}

One can observe in Fig. $5 a$ the computed regular shock interactions in the external inviscid flow that result from the two primary shocks 1 induced by the fins of dissimilar angle as well as arising reflected shocks $1^{\prime}$ and $1^{\prime \prime}$. At the rear shoulders of the fins, the flow undergoes Prandtl-Meyer (PM) expansions. The figure also illustrates the channel throat middle line (TML) along the bottom wall and three cross-section locations at $x / L_{w}=0.24,0.41$, and 0.58 measured from the fin leading edge (marked as $I, I I$, and $I I I$, respectively), in which the corresponding flowfield structures are displayed in Figs. $5 b-5 d$, as well as in Figs. $6 e$ and $7 b$ that depict the experimental surface flow pattern visualization and corresponding computed surface skin-friction lines on the plate, respectively.

As displayed in Fig. $5 b$, the interactions of primary shocks 1 emanating from the fin leading edges with turbulent boundary layer on the plate surface in the vicinity of $7^{\circ}$ left and $11^{\circ}$ right fins in cross section $I$ are similar to that for a 


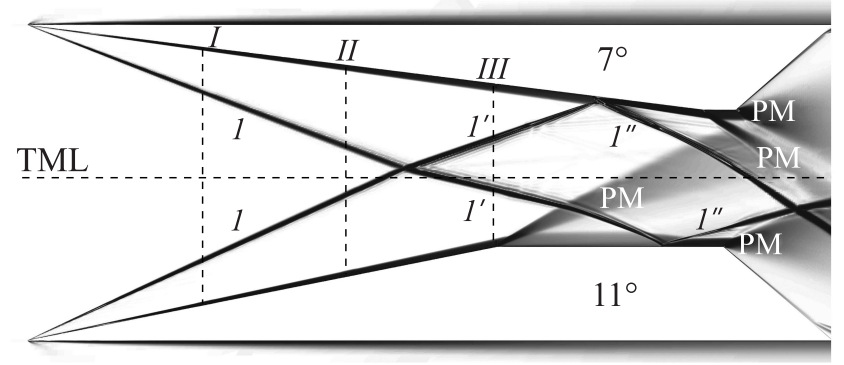

(a)

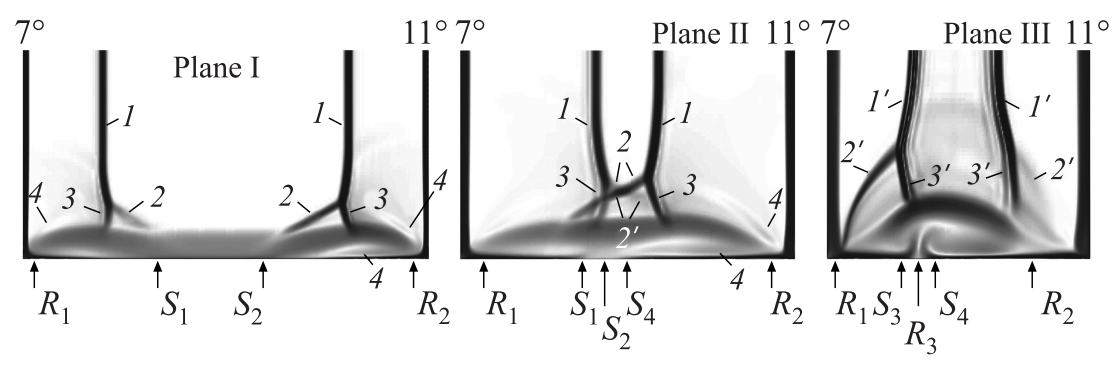

(b)

(c)

(d)

Figure 5 Asymmetric DF crossing shock computed density gradient distributions in the external flow above the plate surface $(a)$ and in cross sections $I(b), I I(c)$, and $I I I(d)$

single fin and the dominant shock feature is the $\lambda$-shock structure comprising the separation shock 2 and the rear shock 3 . Due to the boundary layer separation, a counterrotating vortex pair characterized by low total pressure and low Mach number is formed beneath these $\lambda$ structures. The right vortex of clockwise rotation is larger due to the larger pressure rise in the shock 1 close the right fin compared with the left one. Also observed is a slip (or free shear layer) surface 4 spanning the distance from the triple point to the fin/plate junction. As clearly seen, the shear layer in the vicinity of the right fin penetrates with cross flow to the wall inside the separation zone beneath a detached separated boundary layer.

The flowfield structure in section $I I$ (see Fig. $5 c$ ) displays the stage when the primary shocks 1 have not yet intersected but the forward segments $2^{\prime}$ of the separation shock waves 2 have reflected from one another. In the next cross section $I I I$ (see Fig. $5 d$ ), the primary vortices collide to form a central largescale asymmetric separation zone between the reflected primary shock waves $1^{\prime}$ 


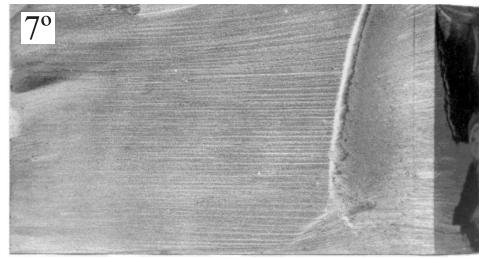

(a)

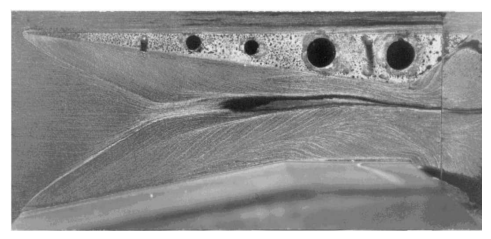

(b)

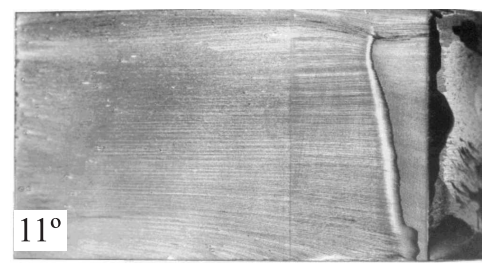

$(c)$
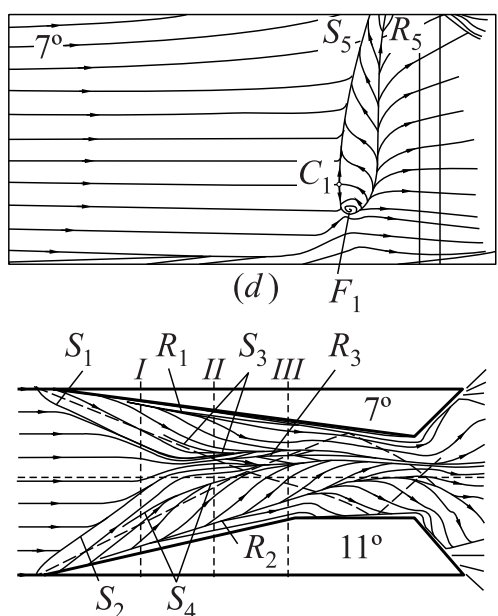

(e)

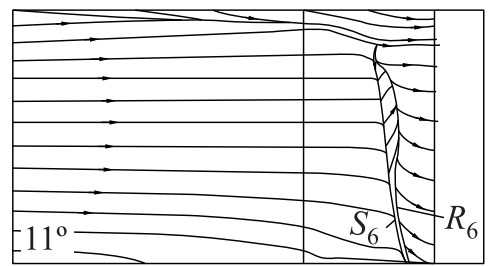

$(f)$

Figure 6 Experimental DF surface flow pattern photographs $(a)-(c)$ and their interpretations $(d)-(f)$

beneath the reflected separation shock waves $2^{\prime}$ and the rear shocks $3^{\prime}$. These are the kinds of features that are not clearly discernible at lower grid resolution (see, e.g., $[13,17,18]$ ).

Experimental surface flow pattern visualization (see Fig. 6) and computed skin-friction coefficient magnitude contours with corresponding skin-friction lines (see Fig. 7) demonstrate good agreement. The incident lines of coalescence (separation) $S_{1}$ and $S_{2}$ on the plate surface emanating from the fin leading edges are evident in the experiment (see Figs. $6 b$ and $6 e$ ) and computation (see Fig. 7e). The computed and experimental separation line angles, measured relative to the incoming free-stream direction, agree within $4 \%$. In accordance with experiment (see Figs. $6 b$ and $6 e$ ), the convergence lines $S_{1}$ and $S_{2}$ form a characteristic "fluid throat" and a streamwise divergence line $R_{3}$ occurs downstream between them. Secondary convergence lines $S_{3}$ and $S_{4}$ are formed due to the interaction of intense secondary flows arising at the fin base from the divergence (reattach- 


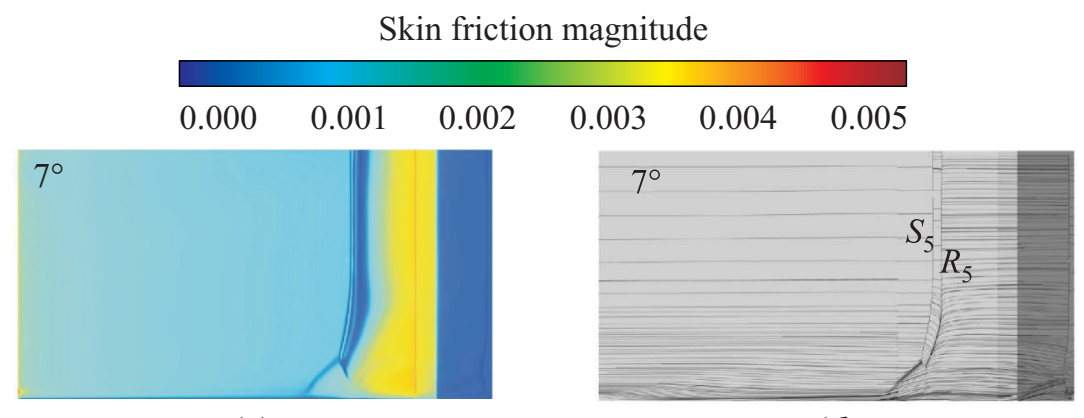

(a)

(d)

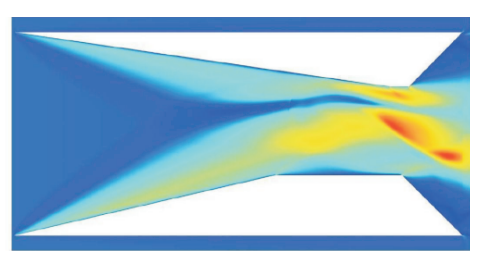

(b)

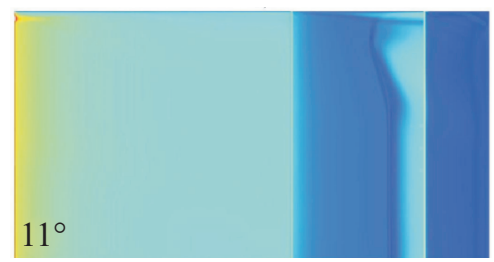

(c)

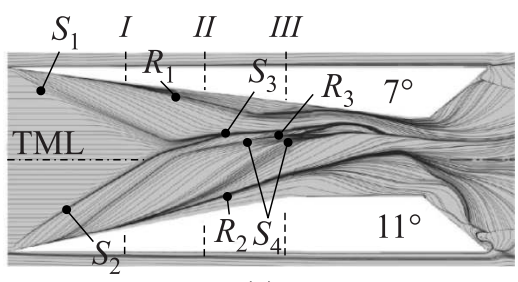

(e)

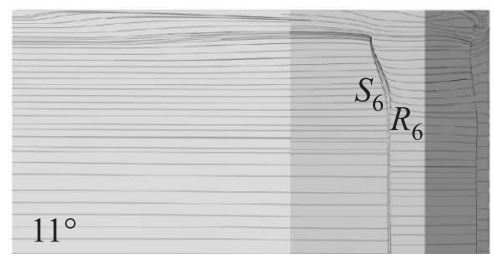

$(f)$

Figure 7 Computed DF surface skin-friction magnitude contours $(a)-(c)$ and surface skin-friction lines $(d)-(f)$.

ment) lines $R_{1}$ and $R_{2}$ with the flow penetrating through the "fluid throat." The line $S_{4}$ observed far upstream in the vicinity of the 11 degree fin as in the case of the single fin test configuration at the same interaction regime (see, e.g., $[2,5]$ ). Existing flow passed through the "fluid throat" without signs of separation was explained by unsteady effects inherent in conditions of incipient intermittent separation [17]. Under these conditions, the employed viscous mineral oil-lampblack mixture for the surface flow pattern experimental visualization does not reflect the influence of the local instantaneous reverse flow, which exists for a short period of time (less than 50\%), and does not register the arising separation point or line in the throat. Because of asymmetry of this configuration, the near-wall flow passing through the "fluid throat" deflects from the channel TML to the region of lower pressure near the fin with shallower 7 degree angle. 
The computed surface skin-friction lines on the plate surface characterize a similar but steady flow. As seen from Fig. $7 e$, the incident primary convergence lines $S_{1}$ and $S_{2}$ in such steady flow coalescence above TML to form a narrow band of skin-friction lines $S_{3}$ that is offset to the left side of the channel and is denoted the left downstream coalescence line. This line represents the surface boundary between the vortices arising in the vicinity of fins. The secondary convergence line $S_{4}$ is associated with secondary separation underneath the vortex of clockwise rotation caused by the spanwise adverse pressure gradient in conditions of the interaction generated by the right 11 degree fin. A streamwise divergence line $R_{3}$ occurs in the "fluid throat" which forms between the convergence lines $S_{3}$ and $S_{4}$ located in the limits of this vortex. Corresponding positions of described specific convergence and divergence lines in the cross sections $I-I I I$ are displayed in Figs. $5 b-5 d$ beneath a realized flowfield structure.

In accordance with Figs. $6 a, 6 c, 6 d$, and $6 f$, the $3 \mathrm{D}$ separation zones bounded by the separation lines $\left(S_{5}\right.$ and $\left.S_{6}\right)$ and corresponding reattachment lines $\left(R_{5}\right.$ and $R_{6}$ ) are formed on the fin internal side surfaces at a certain height from their bases near the region of incidence of the external oblique shock waves $1^{\prime}$ and $1^{\prime \prime}$ (see Fig. $5 a$ ). The separation zone length on the upper $\left(7^{\circ}\right)$ fin in the interaction region with a more intense shock is much larger than on the lower $\left(11^{\circ}\right)$ one. The character of the surface flow pattern on the upper fin surface indicates the existence of singular points: saddle $C$ and focus $F_{1}$. There is no boundary layer separation on the fin surfaces near the plate due to its interaction with forming $\lambda$-shock wave structures. Similar separation and reattachment lines can be seen to be predicted (see Figs. $7 d$ and $7 f$ ), although the degree of separation on the fin walls is lower than what the experiment indicated. This is likely influenced by the enforced fully turbulent RANS treatment in the fin boundary layer. It

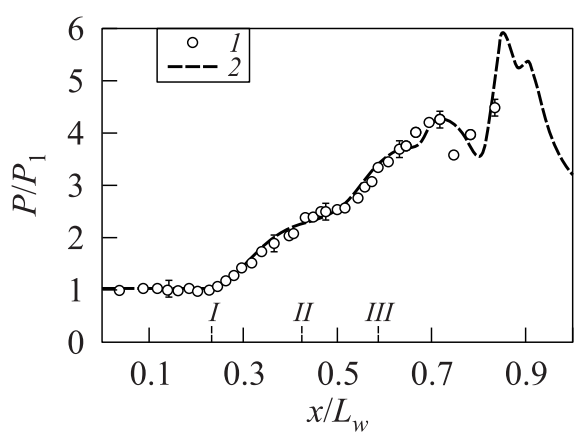

(a)

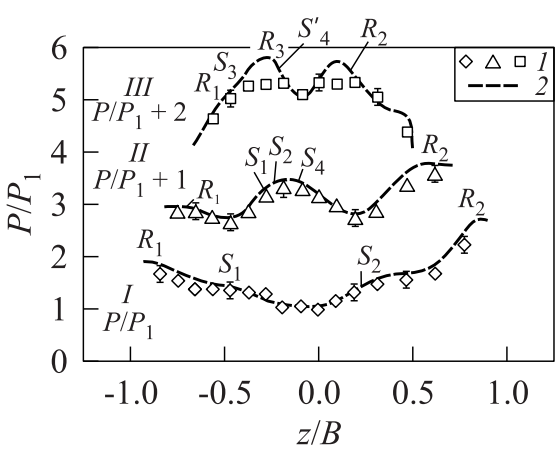

(b)

Figure 8 Double-fin surface pressure distributions on TML $(a)$ and in cross sections $(b): 1-$ experiments; and $2-\mathrm{CFD}$ 
should also be noted that the model choice of setting an inviscid surface at the top of the fin passage (rather than modeling the tunnel test section) resulted in the swept back nature of the separating streamlines not being predicted on the fin surfaces. In accordance with Figs. $7 a-7 c$, the surface skin friction coefficient achieves some locally minimal values along the convergence lines but remains nonzero, as expected for a $3 \mathrm{D}$ ordinary separation [2]. The local maximal $C_{f}$ levels are realized along the divergence lines. As known, regions that exhibit $C_{f}=0$ correspond only to singular separation and reattachment points $[2,6]$.

The computed and experimental surface static pressure distributions on TML and in cross sections $I, I I$, and $I I I$ show close agreement (Fig. 8), although the computation demonstrates 10 percent overestimation of its maximal level in section $I I I$ in the vicinity of divergence lines $R_{2}$ and $R_{3}$. The positions of the convergence and divergence lines displayed in Fig. $7 e$ are marked in Fig. $8 b$ in which the abscissa $z / B$ represents the spanwise relative distance measured from the TML.

\section{TWO CYLINDRICAL BODY FLOW}

\subsection{Flow Conditions and Grid}

The next configuration studied consisted of two cylindrical bodies adjacent to a flat plate (see Fig. 2). The experiments were conducted at the freestream Mach number $\mathrm{M}_{\infty}=4.03$ and the unit Reynolds number $\mathrm{Re}_{1}=55 \cdot 10^{6} \mathrm{~m}^{-1}[19$, $20]$. The adiabatic wall condition was realized in the experiment and the plate turbulent boundary layer thickness was $\delta=1.8-2.0 \mathrm{~mm}$ immediately upstream of the impingement location of the generated bow shock waves on the plate. Two identical conically sharp bodies of revolution $\left(B_{1}\right.$ and $\left.B_{2}\right)$ with two sets of included nose cone angles of $\theta=40^{\circ}$ and $60^{\circ}$, diameter $D=50 \mathrm{~mm}$, and aspect ratio $L_{b} / D=5$ of the body's cylindrical portion were set at the vertical distance $y / D=0.96$ from the plate to their central axes. The details of the flow conditions and test configuration are presented in Table 2. Measurements taken for comparison included schlieren photography of the shock system induced by

Table 2 Experimental and computational conditions for DB test case

\begin{tabular}{cccccccccc}
\hline $\mathrm{M}_{\infty}$ & $\begin{array}{c}\mathrm{Re}_{1}, \\
\mathrm{~m}^{-1}\end{array}$ & $\begin{array}{c}P_{t \infty}, \\
\mathrm{MPa}\end{array}$ & $\begin{array}{c}T_{t \infty}, \\
\mathrm{K}\end{array}$ & $\begin{array}{c}\delta, \\
\mathrm{mm}\end{array}$ & $\begin{array}{c}D, \\
\mathrm{~mm}\end{array}$ & $y / D$ & $L_{b} / D$ & $\theta$ & $\Delta z / D$ \\
\hline 4.03 & $55 \cdot 10^{6}$ & 1.074 & 291 & $1.8-2.0$ & 50 & 0.96 & 5 & $40^{\circ} ; 60^{\circ}$ & $\begin{array}{c}3.0 ; \mathbf{1 . 8} \\
1.4 ; 1.06\end{array}$ \\
\hline
\end{tabular}




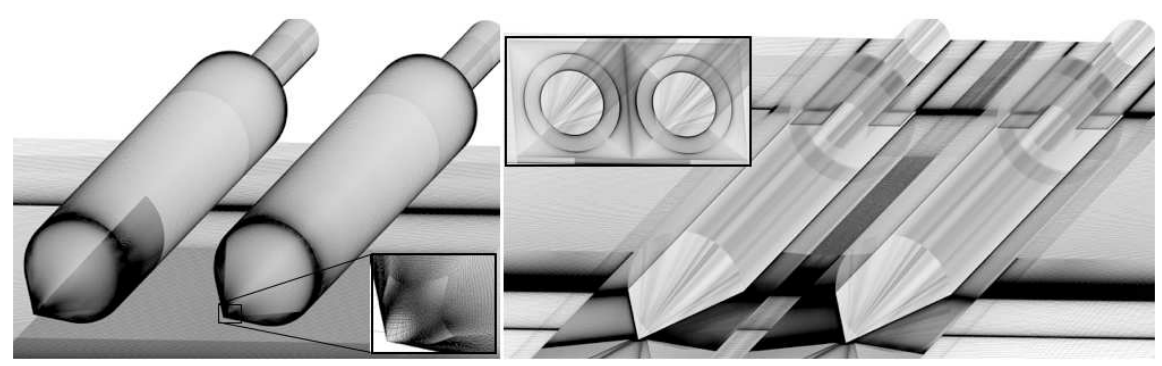

Figure 9 Illustration of DB grid views

the bodies, oil flow visualization on the bodies and plate surfaces, static pressure over the plate surface as well as balance measurements of the bodies' aerodynamic forces.

Prior studies $[15,16]$ focused on a greater number of configurations, analyzed with a base grid resolution of 60 million cells that modeled one symmetric half of the flow at different distances between the bodies axis: $\Delta z / D=3.0,1.8,1.4$, and 1.06. The 1.8 diameter spacing is given greater focus herein, at nose cone angles of $40^{\circ}$ and $60^{\circ}$. In these two cases, a grid containing half a billion grid cells and one billion grid cells were employed, respectively, explicitly modeling both bodies rather than using a symmetry plane boundary condition, to maximize the resolution of the shock-shock and shock-boundary layer interaction regions in the vicinity between the bodies and plate, as well as on the outside of the bodies (see, e.g., Fig. 9).

\subsection{Results}

Figures 10-12 display computed shock wave structures surrounding the bodies, respectively, in vertical and horizontal centerline cutting plane views and in some selected cross sections $I, I I$, and $I I I$ at $\theta=40^{\circ}, \Delta z / D=1.8$. Experimental photographs of surface flow patterns on the body $B_{1}$ are displayed in Figs. $10 a$ and $11 b$, and Fig. $11 a$ provides an experimental schlieren photograph for comparison with predicted shock structures and surface skin-friction coefficient magnitude contours (see Figs. $10 b$ and 11c) as well as surface skinfriction lines (see Fig. 11b). The results agree well with experiment in terms of shock angles, bow shocks $1,1^{\prime}$ and $A, A^{\prime}$ extending from their regular interaction as well as additional reflected shocks $B, B^{\prime}$ between the bodies (see Figs. $11 a$ and $11 b$ ). Prandtl-Meyer expansion waves extend from the cone/cylinder junction. One can observe the presence of separation-induced shock wave $1_{S}$, its continuation $2^{\prime}$, and terminal shock $1_{R}$ in the reattachment region beneath 


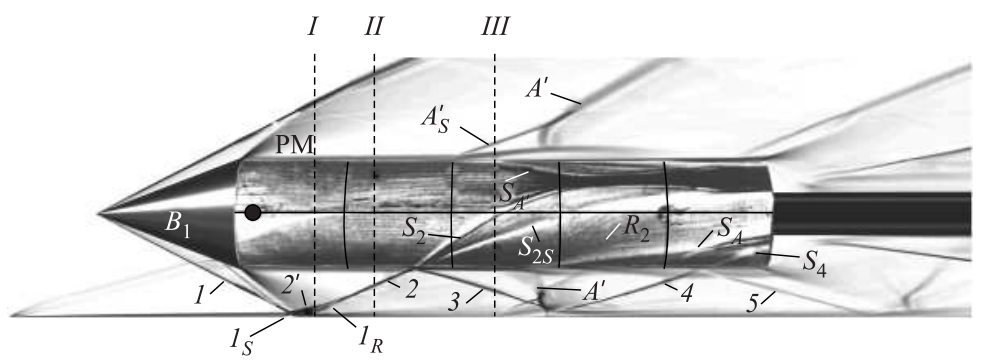

(a)

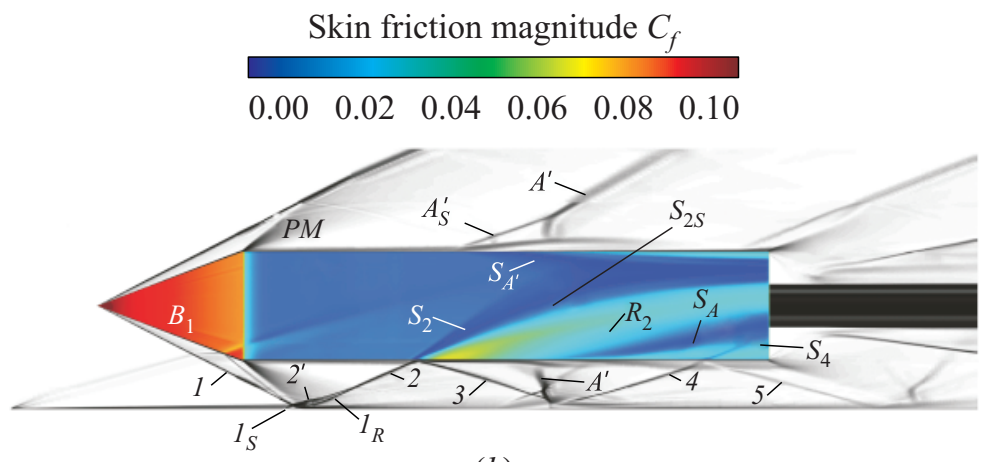

(b)

Figure 10 Computed shock wave structures in body centerline vertical cutting plane with experimental surface flow pattern $(a)$ and predicted surface skin friction $(b)$ on outside surface of body $B_{1}$ for $\theta=40^{\circ}, \Delta z / D=1.8$.

the body (see Fig. 10). Upon merging, shocks $2^{\prime}$ and $1_{R}$ form a single reflected shock wave 2 , which reaches the body surface and reflects again between the body and plate as a system of successive reflected shock waves 3 , 4 , and 5 . The shock wave 2 diffracting around the body stimulates a $3 \mathrm{D}$ separation zone on its outside surface between the lines $S_{2}$ and $R_{2}$ with arising secondary separation along the convergence line $S_{2 S}$, and diffracting shock 4 causes the separation line $S_{4}$. The reflected shocks 2 and 4 induce two corresponding horseshoe-shaped separation lines $S_{2}$ and $S_{4}$ on the body underside surface shown in Fig. $11 b$. The shock wave $A^{\prime}$ diffracts around the body and stimulates the separation line $S_{A}$ with corresponding separation-induced shock wave $A_{S}^{\prime}$ which spread from the internal surface around to the top and bottom surfaces. Computed skin friction distributions and skin-friction lines on the body surfaces (see, e.g., Figs. 10b, 11b, and 11c) demonstrate good correspondence with the experimental surface flow pattern. The local $C_{f}$ minimums 


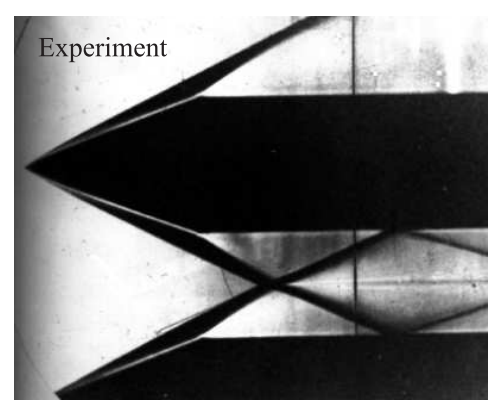

(a)

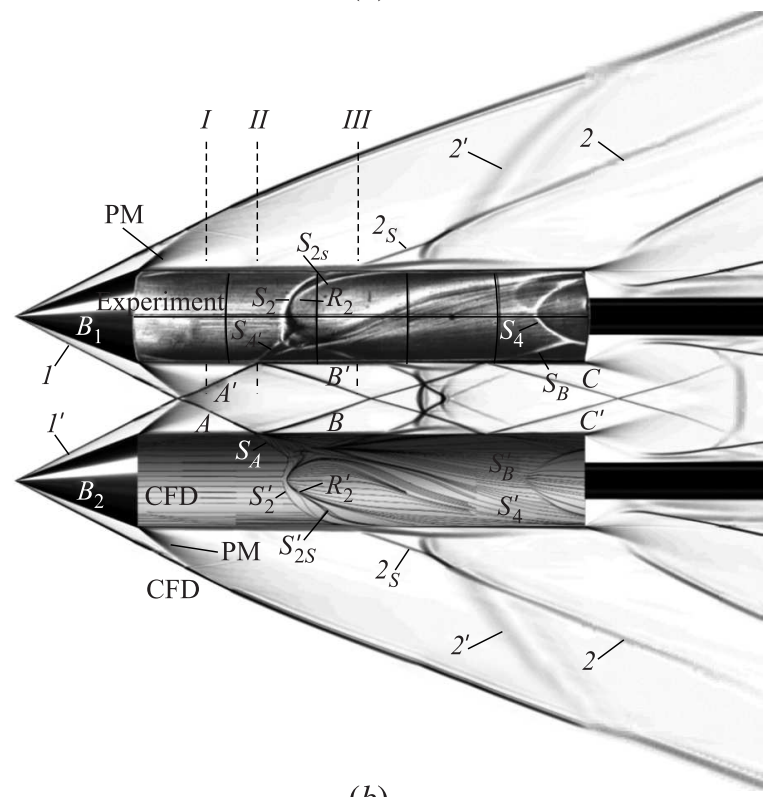

(b)
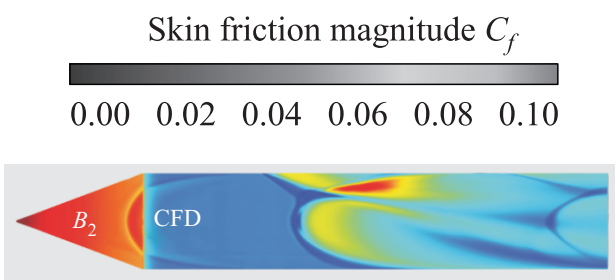

(c)

Figure 11 Experimental schlieren photograph $(a)$ and computed shock wave structures in horizontal cutting plane through centerlines of bodies with experimental and predicted surface flow pattern $(b)$, and skin friction $(c)$ on bottom surface of bodies for $\theta=40^{\circ}, \Delta z / D=1.8$. 


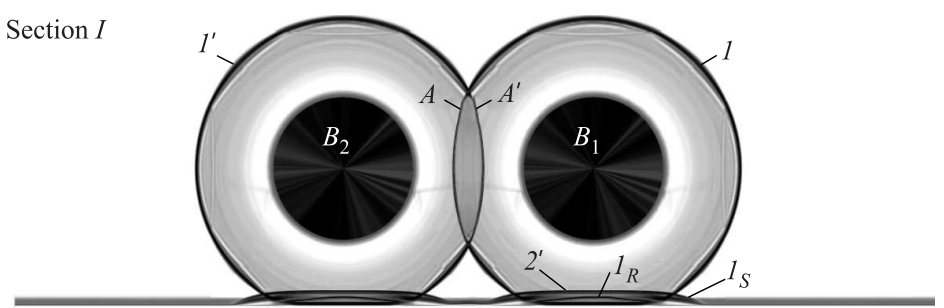

(a)

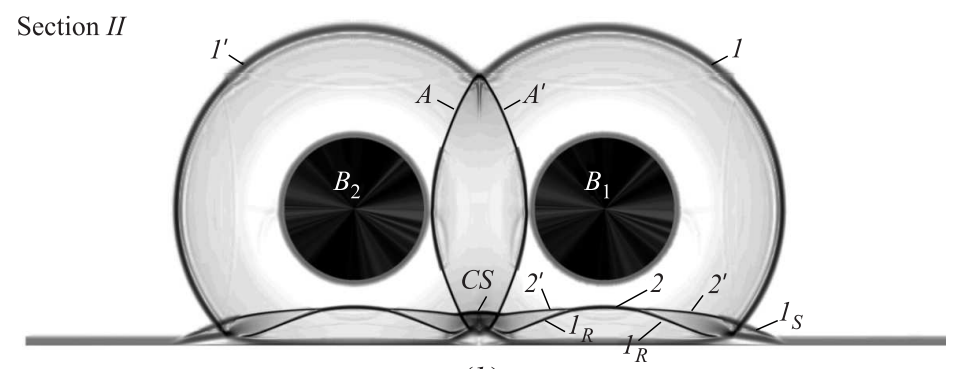

(b)

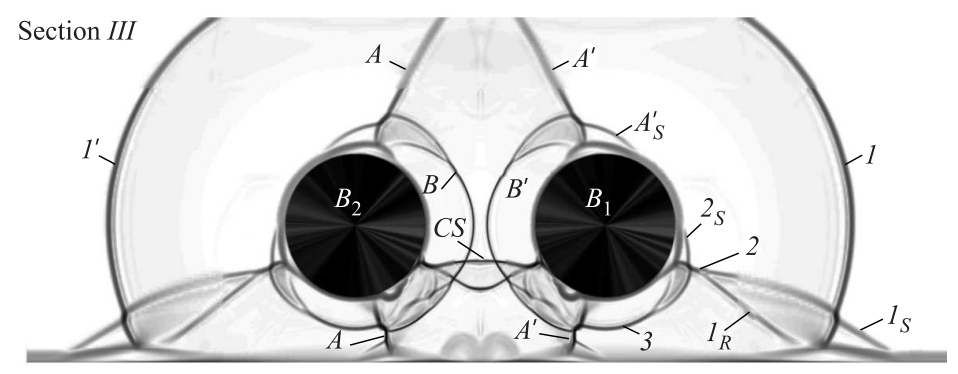

(c)

Figure 12 Series of longitudinal cutting plane views of predicted 3D shock structures for $\theta=40^{\circ}$ and $\Delta z / D=1.8$

arise along the convergence lines and the maximal values appear along the divergence lines. In accordance with Figs. $11 a$ and $11 b$, the crossing bow shocks $\left(A\right.$ and $\left.A^{\prime}\right)$ and reflected shocks $\left(B\right.$ and $\left.B^{\prime}\right)$ intersect the body inside surfaces and, respectively, induce the horseshoe-shaped separation lines $S_{A}, S_{A^{\prime}}$ and $S_{B}$, $S_{B^{\prime}}$.

Predicted evolution of shock structures in cross sections $I, I I$, and $I I I$ (marked in Figs. 10 and 11) are shown in Fig. 12. In accordance with these data, a distinct 3D separation zone arises on the plate not only along the external boundary of the interaction region beneath the separation-induced shock 
wave $1_{S}$ and its symmetrical counterpart but also between the bodies beneath the central shock $C S$. In section $I I$, the crossing bow shocks $A^{\prime}, A$ as well as reattachment shocks $1_{R}$ surround the central separation zone. In section $I I I$, the shocks $A^{\prime}, A$ diffracting around the bodies stimulate corresponding reflected shocks $B, B^{\prime}$ from their internal surfaces. In this section, the shock waves $A^{\prime}$ and 2 as well as their symmetrical counterparts stimulate 3D separation zones on the body surface which are located beneath the $\lambda$-shock structures including the leading separation-induced shock waves $A_{S}^{\prime}, 2_{S}$. Similarly, the $\lambda$-shock structures arise in the vicinity of reflected bow shock waves $A^{\prime}$ and $A$ penetrating beneath the bodies which stimulate boundary layer separation from their underside surfaces and on the plate surface. The phenomena of complex crossing shock wave structures observed in sections $I, I I$, and $I I I$ briefly described herein explains the presence of additional shock waves (e.g., $A_{S}^{\prime}, 2_{S}$ ) seen in Figs. 10 and 11.

Comparison of experimental plate surface flow pattern visualization (Figs. 13a-13c) and computed skin-friction coefficient magnitude contours with corresponding skin-friction lines (Figs. 13d-13f) demonstrate good agreement. Detailed description of the properties and topology observed in experiment at $\theta=40^{\circ}$ and $\Delta z / D=1.8$ was presented in [19]. An external boundary of the 3D separated zone beneath the bodies is limited by the primary convergence (separation) lines $S_{1}, S_{1}^{\prime}$ (see Fig. $13 b$ ). Two symmetric saddle separation points $C_{1}$ and $C_{1}^{\prime}$ located in the apex of these convergence lines and corresponding symmetric nodal reattachment points $N_{1}$ and $N_{1}^{\prime}$ arise in the apex of the primary divergence (reattachment) lines $R_{1}$ and $R_{1}^{\prime}$. The secondary convergence $S_{2}$ and divergence $R_{2}$ lines and their symmetric counterparts are observed between the primary convergence and divergence lines. The downstream convergence lines $S_{3}$ and $S_{3}^{\prime}$ are initiated by reflected shock waves $3,3^{\prime}$ beneath the bodies. Additionally, the secondary convergence and divergence lines $\left(S_{4}\right.$ and $\left.R_{4}\right)$ and their symmetric counterparts are observed in the flow behind the lines $S_{3}$ and $S_{3}^{\prime}$. The central separation zone forms downstream of the cross separation line $S_{0}$ that arises in the vicinity of the symmetry line $O_{S}$ on the plate corresponding to the vertical symmetry plane between the bodies. Details of the surface flow pattern topology are depicted in Fig. $13 c$. The central saddle point of separation $C_{0}$ arises in the middle of the line $S_{0}$ which is bounded by symmetric nodal separation points $N_{2}$ and $N_{2}^{\prime}$. The central separation zone is limited downstream by symmetric primary divergence lines $R_{1}, R_{1}^{\prime}$ which meet at the central saddle point $C_{3}$. Intense reverse flow propagates from the point $C_{3}$ to $C_{0}$. The oil flow forms two nearly symmetric large-scale foci $\left(F_{1}\right.$ and $\left.F_{1}^{\prime}\right)$. As was concluded in [19], dual side saddle points $C_{2}$ and $C_{2}^{\prime}$ emerge behind the nodes $N_{2}$ and $N_{2}^{\prime}$. Located downstream of the central separation zone are symmetric convergence lines $S_{A}^{\prime}$ and $S_{A}$ and corresponding divergence lines $R_{A}^{\prime}$ and $R_{A}$ (see Fig. 13b) which are initiated by reflected bow shock waves $A^{\prime}$ and $A$ propagating beneath the bodies. 


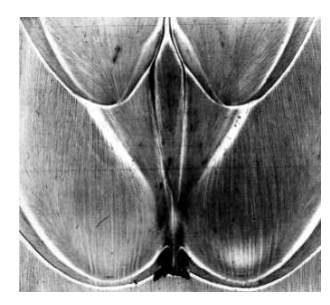

(a)

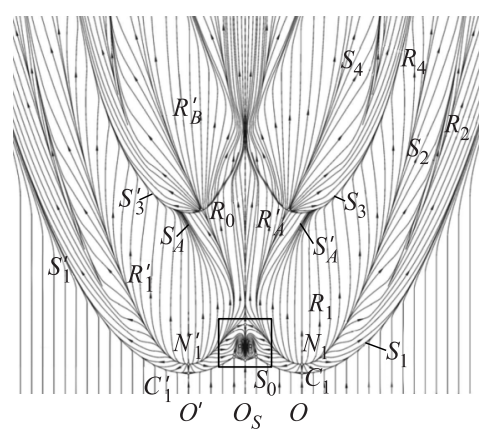

(b)

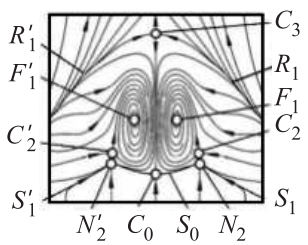

(c)

Skin friction magnitude

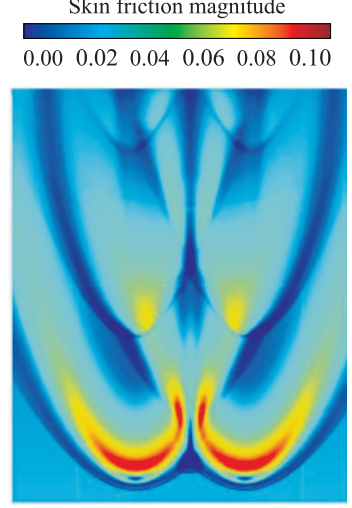

(d)

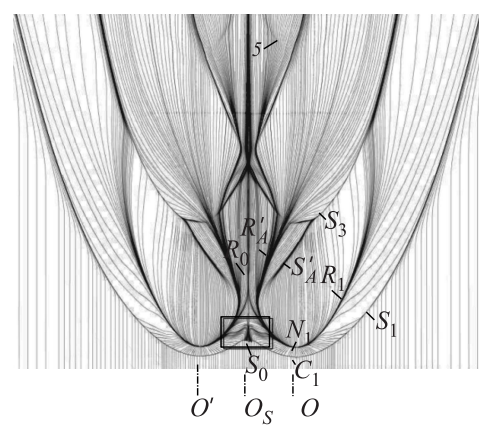

(e)

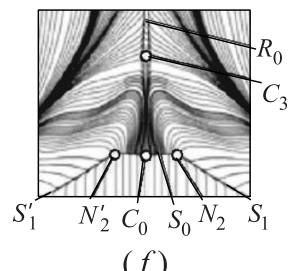

Figure 13 Experimental photograph of plate surface flow pattern visualization $(a)$ and its interpretation $(b)$ and $(c)$ and predicted surface skin friction $(d)$ with corresponding surface skin-friction lines $(f)$ and $(e)$ beneath DB for $\theta=40^{\circ}$. 


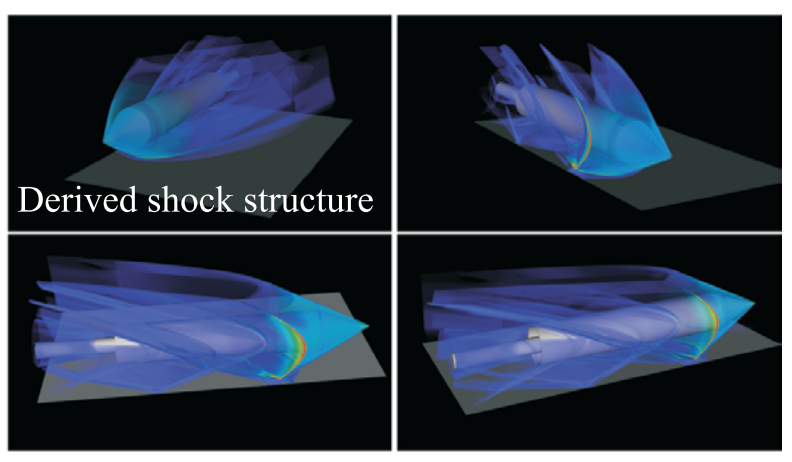

\section{(a)}

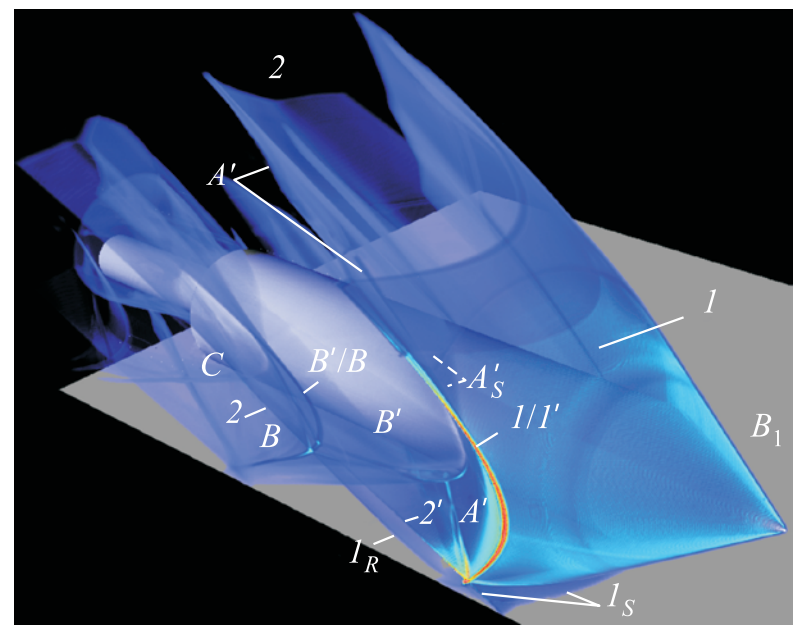

(b)

Figure 14 Volume rendering of predicted DB 3D shock structure for $\theta=40^{\circ}$ and $\Delta z / D=1.8$.

Although the predicted topology of plate surface skin-friction lines (see Figs. $13 e$ and 13f) in general is very similar with that observed in experiment, two marked distinctions can be noted. The two large-scale foci $\left(F_{1}\right.$ and $\left.F_{1}^{\prime}\right)$ and two side saddles $\left(C_{2}\right.$ and $\left.C_{2}^{\prime}\right)$ behind the symmetric nodal separation points $N_{2}$ and $N_{2}^{\prime}$ have not been predicted in the surface flow (see Fig. 13f). Such a difference could possibly be explained by an accumulation of oil curling up around the foci centers in experiment which cannot leave the surface with the air flow. Thus, the large-scale foci and side saddles could be considered as phenomena typical of the motion of viscous oil film on the plate surface but not the clean 
air flow which in accordance with computation leaves the surface in the nodal (focus-type) separation points $N_{2}$ and $N_{2}^{\prime}$. In addition, the secondary separation phenomenon associated with observed pairs of secondary convergence and divergence lines $S_{2}, R_{2}$ and $S_{4}, R_{4}$, (see Fig. $13 b$ ) was not predicted. This could be explained by some overestimation of the turbulence level in the interaction region by the employed SA model as was also demonstrated by computations of different 3D SWTBLI with other turbulence models [2]. However, this can be also explained by the choice of grid distribution that was refined strongly between the bodies but less so in the outside of the interaction regions [16]. Despite some described differences, computed surface skin-friction magnitude panorama (see Fig. $13 d$ ) demonstrates good agreement with experimental surface flow pattern visualization (see Fig. 13a). The predicted last reflected shock waves 5 beneath the bodies (see Fig. 10) stimulate specific symmetric regions 5 with a bending of surface skin-friction lines without signs of separation (see Fig. 13e) and the corresponding last pair of symmetric horseshoe-shaped regions of reduced $C_{f}$ values observed in Fig. $13 d$.

In Fig. 14, the 3D shock structure predicted using one billion grid cells is shown. Here, a derived "shock" data field was created by multiplying the three Cartesian components of density gradient and velocity, summing them, and dividing by magnitude of velocity. This produces a scalar that tends to clearly show 3D shock structures when employing volume rendering (or volume ray casting). The visualization shown was produced using the VisIt open source interactive parallel visualization and graphical analysis tool. Adjacent to the 3D volume rendered views, a magnified view is provided, with the major shock structures labelled. One can gain a much better appreciation of the $3 \mathrm{D}$ shock structures by viewing this representation, in addition to the earlier two-dimensional (2D) cuts that were discussed, and comparing the earlier $2 \mathrm{D}$ projections with the actual intersecting and reflecting conical 3D structures. For instance, one can observe the behavior of the lambda shock foot region of the primary intersection of the bow shocks 1 and the plate with arising separation shock front $1_{S}$ as well as the second reflected bow shock $A$ diffracting around the body with corresponding separation shock $A_{S}^{\prime}$. One can also see the $3 \mathrm{D}$ nature of the first reflected separation $2^{\prime}$ and reattachment $1_{R}$ shocks as well as reflected shocks $A^{\prime}, B^{\prime}, B$, and $C$ between the bodies.

Corresponding predicted 3D streamlines in the symmetry plane and in the primary separation zone beneath the left body $B_{2}$ characterize complex flowfield structure arising above plate surface (Fig. 15). The boundary layer that separates along the primary separation line $S_{1}^{\prime}$ beneath the body and the emerging vortex contain fluid that sweeps spanwise to separate on the downstream side of the primary separation line and penetrate into the central separation zone in the vicinity of the symmetry plane. The fluid separating in the saddle point $C_{0}$ is deflected downstream of the reattachment shock $1_{R}$. 


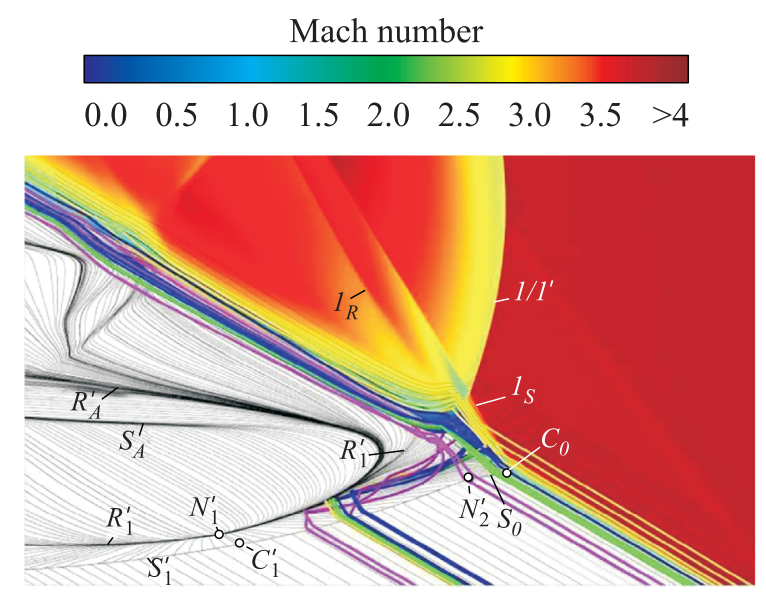

Figure 15 View of predicted symmetry plane flow and 3D separating streamlines in the primary separation zone between the bodies and beneath the left body $B_{2}$ for $\theta=40^{\circ}$ and $\Delta z / D=1.8$.

Figure 16 compares an experimental schlieren photograph of the shock wave structure and inside body surface flow pattern photograph $(a)$ with computed shock wave structure and surface skin-friction lines $(b)$ at the higher body nose cone angle of $\theta=60^{\circ}$ for $\Delta z / D=1.8$. Corresponding comparison for the body underside surface flow pattern and crossing shock waves structures on a horizontal cutting plane is demonstrated in Fig. 17, and predicted evolution of the Mach number magnitude contours and shock structures in cross sections $I, I I$, and $I I I$ is shown in Fig. 18. The main notations in the figures correspond to that used for the previous test case. The comparisons demonstrate good agreement of predictions with experimental data. In accordance with these results, increasing the bow shock wave strength and its deflection angle stimulate more pronounced separations zones as well as their shift in the upstream direction, compared with $\theta=40^{\circ}$ case.

In accordance with experiment, the central saddle point of separation $C_{0}$ observed on the plate surface at $\theta=60^{\circ}$ is displaced further towards the freestream by intense reverse flow propagating from the attachment saddle point $C_{3}$ arising in the region of elevated pressure, which is followed by a growth of the extension of the advancing transfer separation line $S_{0}$ bounded by symmetric nodal (focus-type) separation points $N_{2}$ and $N_{2}^{\prime}$ (Figs. $19 a$ and 19b). The distance between the saddle points of separation $C_{1}, C_{1}^{\prime}$ and the corresponding nodal points of reattachment $N_{1}, N_{1}^{\prime}$ beneath the bodies is also increased. Note some flow asymmetry downstream of the central separation zone observed in the experimental traces which leads to the singular points $C_{0}, C_{3}, C_{0}^{1}$, and $N_{0}$ on 


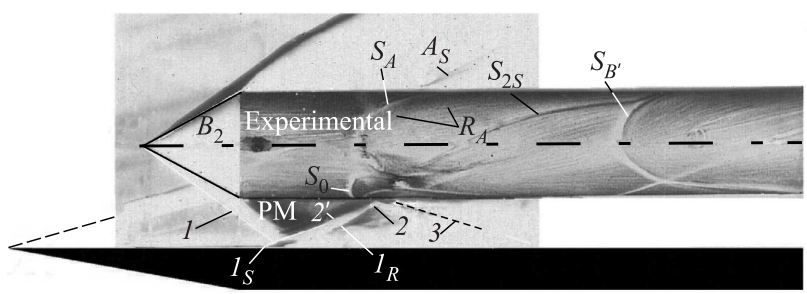

(a)

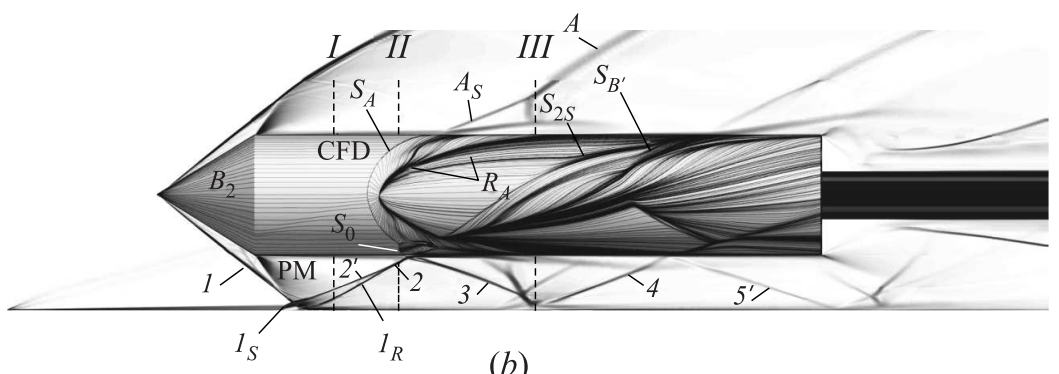

(b)

Figure 16 Experimental $(a)$ and computed $(b)$ shock wave structures in body centerline vertical cutting plane and surface flow pattern on inside surface of body $B_{2}$ for $\theta=60^{\circ}$ and $\Delta z / D=1.8$

the surface being located at small displacements from the central line $O_{S}$, as shown in Fig. 19a. In accordance with [19, 20], such asymmetry could be due to small deformations of the test model. The surface static pressure distribution panorama in the interaction region is displayed in Fig. 19c. The pressure maxima $A$ and $B$ correspond to the symmetric nodes $N_{1}$ and $N_{1}^{\prime}$ on the plate beneath the bodies $B_{1}$ and $B_{2}$, respectively, and the central maximum $C$ arises at the central saddle point $C_{3}$. Details of observations in experimental surface flow pattern topology and pressure distribution with formed additional symmetric $(D, E)$ and central $(M)$ maxima are discussed in $[19,20]$.

The predicted topology of surface skin-friction lines (Figs. 20a and 20b) and pressure distribution (Fig. 20c) in general are very similar with that observed in experiment. However, the central separation zone between the bodies is smaller as compared to experimental observations in Figs. $19 a$ and $19 b$. It is natural that some experimentally observed asymmetry in surface flow pattern would not be predicted, due to the imposed geometrical symmetry of the numerical model. It is significant that the incipient secondary convergence lines $S_{2}$ and $S_{4}$ begin to be predicted, though they are not as distinct as in experiment. The predicted static pressure coefficient distribution (Fig. 20c) is seen to reproduce the pressure field 


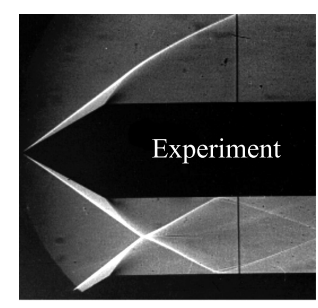

(a)

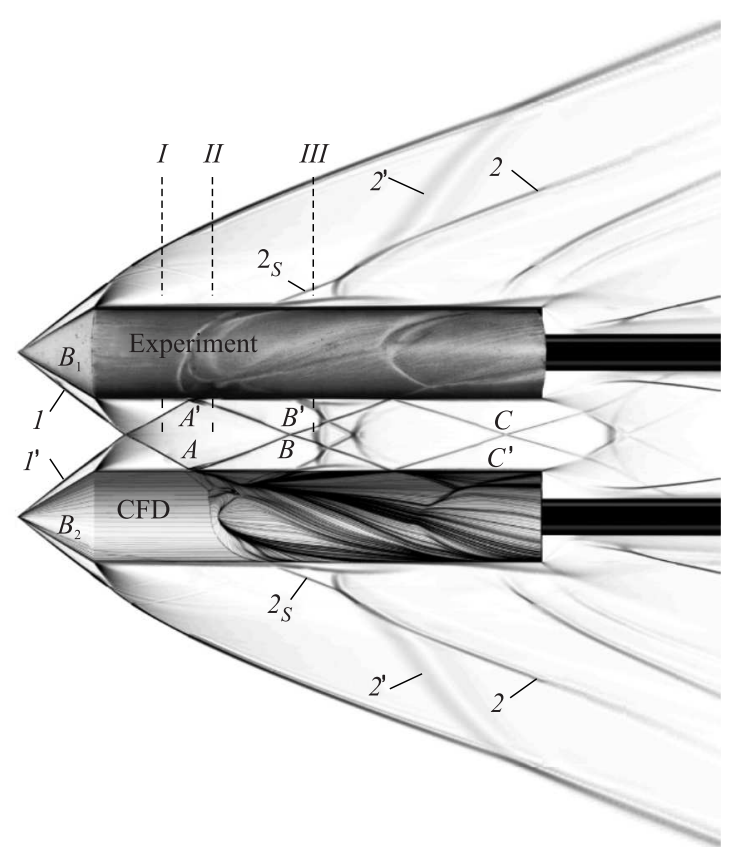

(b)

Figure 17 Experimental schlieren photograph $(a)$ and computed shock wave structures in horizontal cutting plane through centerlines of bodies with experimental and predicted surface flow pattern $(b)$ on bottom surface of bodies for $\theta=60^{\circ}$ and $\Delta z / D=1.8$

observed in experiment quite well. As in previous test case at $\theta=40^{\circ}$, the two large-scale foci $\left(F_{1}\right.$ and $\left.F_{1}^{\prime}\right)$ and two side saddles behind the nodes $N_{2}$ and $N_{2}^{\prime}$ (see Fig. 19a) have not been predicted in the surface flow (see Fig. 20a).

As was concluded above, the large-scale foci characterize the motion of accumulated viscous oil film on the plate surface in experiment. This appears to be supported by Fig. 21 where computed 3D air streamlines can be seen to curl up into a vortex around the nodal (focus-type) point $N_{2}^{\prime}$ at a small distance off the surface (see dash-dot lines). The flow topology with similar pair of the nodal (focus-type) points and the vortices arising around them have been also predicted in [21] in the central separation zone between symmetric $\left(18^{\circ} \times 18^{\circ}\right.$ and $23^{\circ} \times 23^{\circ}$ ) DFs at $\mathrm{M}_{\infty}=5$. As shown in Figs. $21 a$ and $21 b$, an intensive vortex arises in the separation zone between the primary convergence $\left(S_{1}^{\prime}\right)$ and divergence $\left(R_{1}^{\prime}\right)$ lines and the fluid in the vortex spreads to the symmetry plane where the flow separates again along the side convergence line $S_{S}^{\prime}$ that forms in the central separation zone (see Fig. $21 b$ ) with reattachment downstream along 


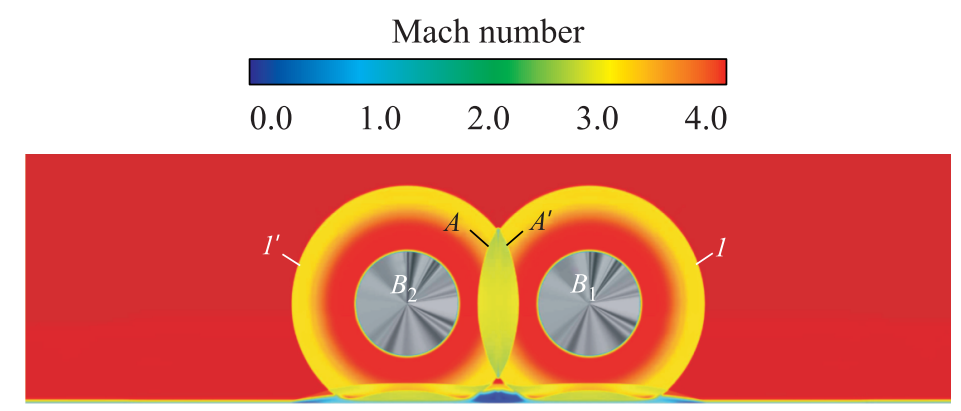

(a)

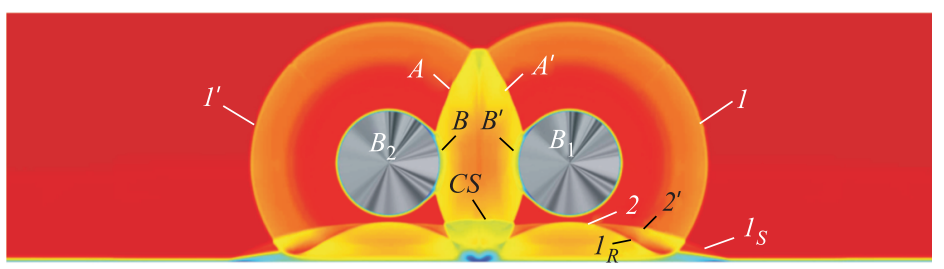

(b)

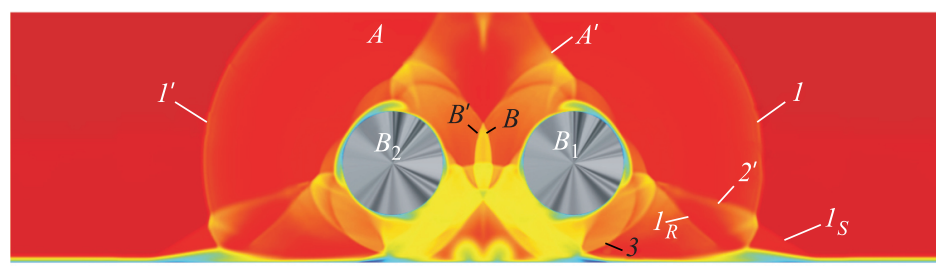

(c)

Figure 18 Series of longitudinal cutting plane views of predicted Mach numbers and shock structures for $\theta=60^{\circ}$ and $\Delta z / D=1.8$ : (a) section $I ;(b) I I$; and $(c)$ section $I I I$.

the central divergence line $R_{0}$. The line $S_{S}^{\prime}$ and its symmetric counterpart $S_{S}$ are shown also in Fig. 20b. The central divergence line $R_{0}$ spreads in the upstream and downstream directions from the saddle point $C_{0}^{\prime}$.

Figure 22 compares predicted body aerodynamic forces coefficients $\left(C_{x}, C_{y}\right.$, and $C_{z}$ ) for a series of interbody distances with their experimental values from [20]. One can observe approximately constant drag coefficient $C_{x}$ and a gradual $C_{y}$ and $C_{z}$ increasing for the 60 degree nose angle case as interbody distance decreases, up to $\Delta z / D=1.4$. Such behavior is explained by a gradual development of the static pressure and 3D separation zone only on the cylindrical surfaces of the bodies at such conditions (Figs. 23a-23c). The regular 


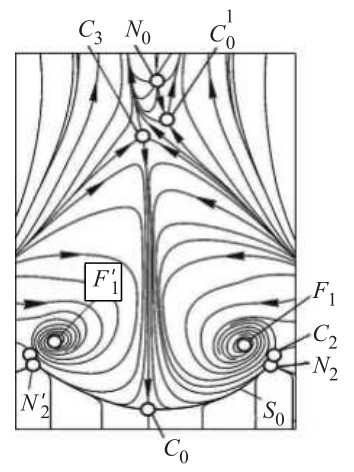

(a)

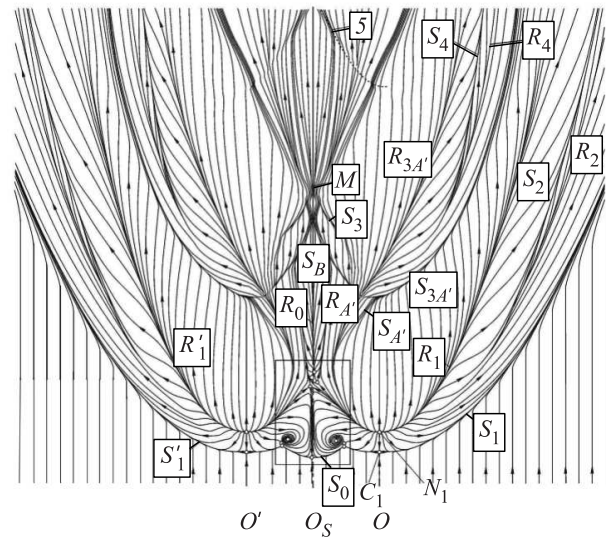

(b)

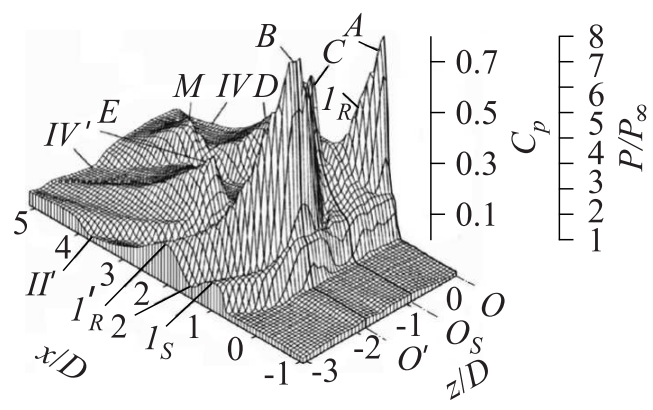

(c)

Figure 19 Experimental plate surface flow pattern $((a)$ and $(b))$ and static pressure distribution $(c)$ beneath $\mathrm{DB}$ for $\theta=60^{\circ}$ and $\Delta z / D=1.8$

crossing bow shock waves structures were predicted and observed in experiment at such conditions $[15,16]$. In accordance with the computations and experiment, these flow regimes are followed by the appearance of a small Mach stem between the bodies as the interbody distance is reduced to $\Delta z / D=1.4$. The cardinal reconstruction of separated flow occurs with decreasing distance between the bodies to $\Delta z / D=1.06$ at which a distinctly formed Mach stem indicates a global "unstart" phenomenon in the limited space between the bodies and plate. The separation zone penetrates upstream to the conical nose surface at such conditions (Fig. 23d) and stimulates significant additional rise of all the aerodynamic coefficients (see Fig. 22). One can also observe a marked decrease in the force coefficients for the 40 degree nose angle at $\Delta z / D=1.8$ compared to the corresponding values for $\theta=60^{\circ}$ case. 


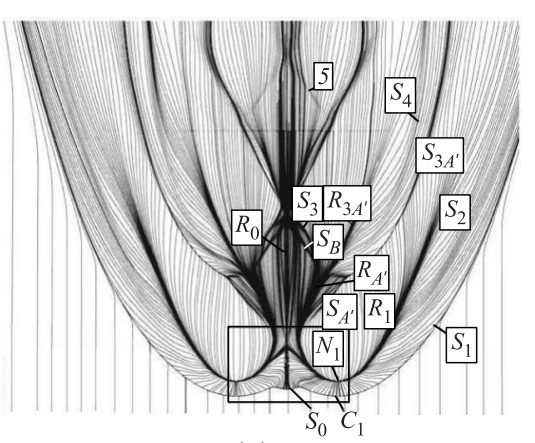

(a)

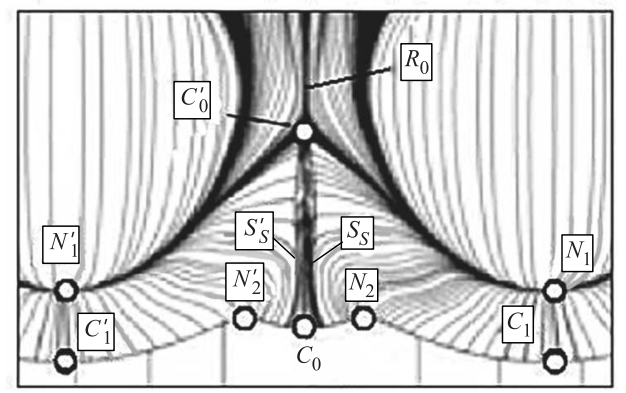

(b)

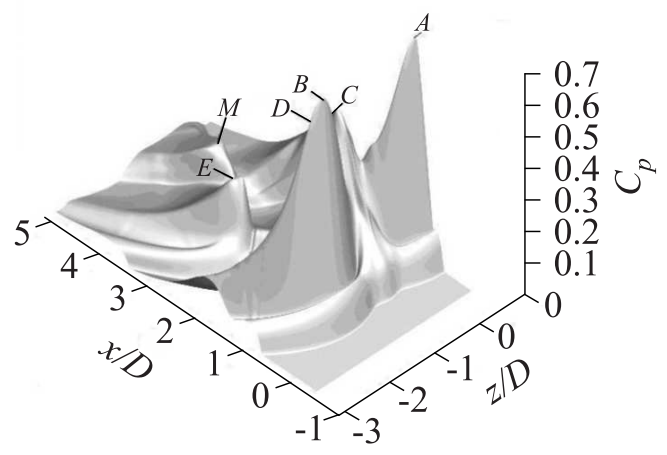

(c)

Figure 20 Computed surface skin-friction lines $((a)$ and $(b))$ and surface pressure coefficient distribution $(c)$ beneath $\mathrm{DB}$ for $\theta=60^{\circ}$ and $\Delta z / D=1.8$

\section{TRANSONIC FAN WITH WALL JET TIP INJECTION}

\subsection{Motivation}

The prior test cases that have been presented focus on detailed analysis of shock wave / turbulent boundary layer interaction and prediction of these 3D phenomena. In the present case, the focus is on a shock wave / turbulent boundary layer interaction-driven loss mechanism in transonic compressors and the potential reduction of such induced distortion using boundary layer flow control is discussed. The state of flow at the shroud endwall or 'casing' that exists at the outer radius of transonic and supersonic compressors (see Fig. $3 a$ ) has been identified in prior literature as being a critical area where low momentum and ultimately separated 


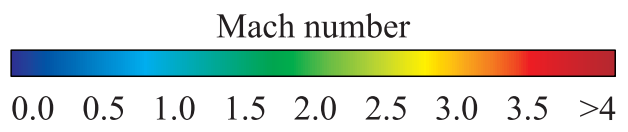

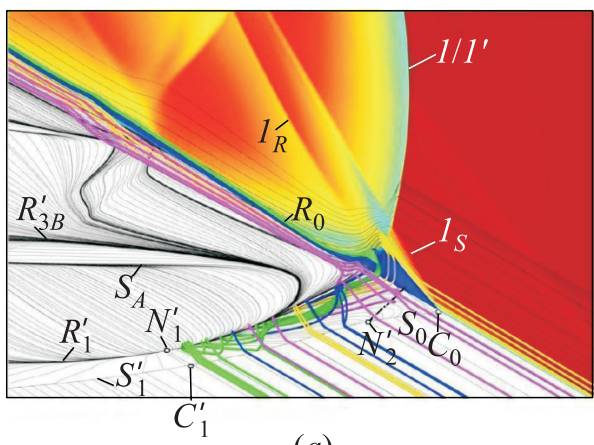

(a)

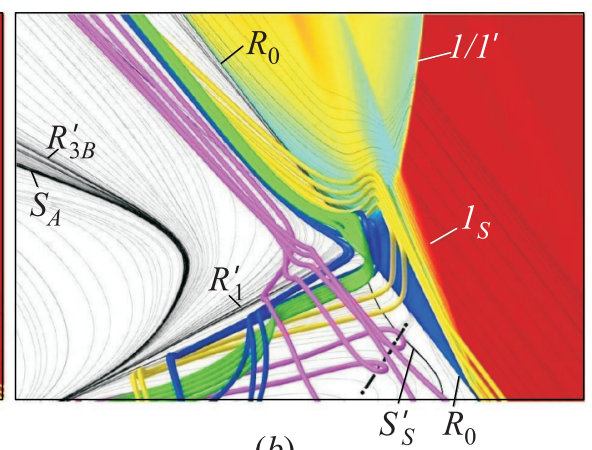

(b)

Figure 21 View $(a)$ and magnified view $(b)$ of predicted symmetry plane flow and 3D separating streamlines in the primary separation zone between the bodies and beneath the left body $B_{2}$ for $\theta=60^{\circ}$ and $\Delta z / D=1.8$.

flow acts as a primary driver of compressor stall. Flow separation in the region of the blade (particularly suction surface) / shroud intersection (see Fig. $3 b$ ) is driven by the state of the incoming boundary layer (e. g., boundary layer momentum thickness, shape factor, local total pressure), the leading edge and passage shock wave strengths, and presence of tip clearance flow. The critical nature of the state of the casing endwall flow has been highlighted in previous compressor literature. In [22], the authors noted the development of structures inside the casing boundary layer at near-stall operating conditions, that stall initiates very close to the casing, and that absence of tip clearance gap increased stall margin by $20 \%$. Similar findings were made in [23] in the context of studies focusing on the spike mode of stall. Compressors in operation today, such as those present in aircraft gas turbines, are restricted to the transonic regime. Rotating supersonic compression still largely represents a field of research, due to the dominating influence of passage shock waves in such configurations. A review [24] of the past 60 years of attempts by researchers to develop supersonic compressors identified two key areas that have recently been advanced, which offer the modern designer of supersonic compressors additional tools to reach potential success. The advent of modern CFD techniques was identified, and sophisticated boundary layer flow control techniques (what they referred to as "surge control systems" which consist of wall jet-type fluid injection flow control devices installed at the casing).

In addition to the significant degree of unsteadiness that can inherently exist in a separating shock wave- boundary layer interaction (SWBLI) flow, in such ro- 


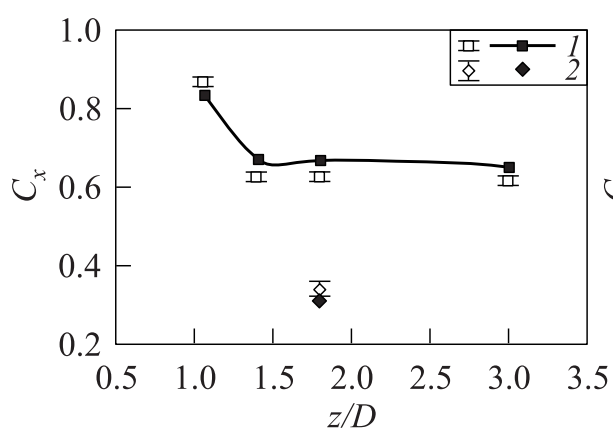

(a)

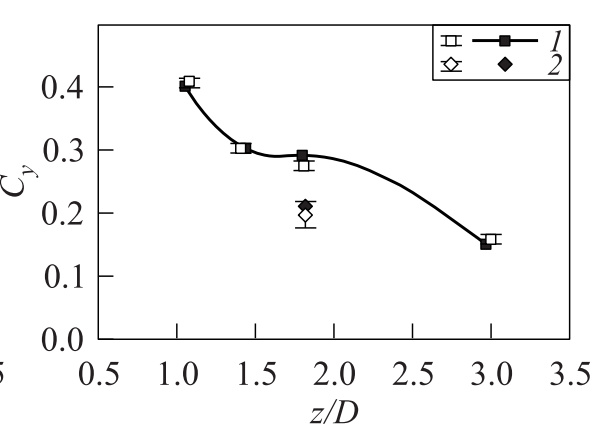

(b)

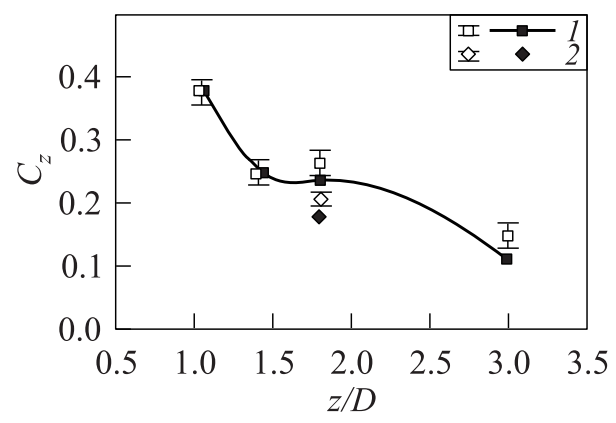

(c)

Figure 22 Comparison of predicted (filled symbols and curves) aerodynamic force coefficients with balance measurements (empty symbols) for $\theta=60^{\circ}(1)$ and $40^{\circ}(2)$ : (a) drag component; $(b)$ lift component; and $(c)$ side force component

tating machinery there is another primary source of aerodynamic time-varying behavior that arises from the interaction of a rotating set of turbomachinery blades passing a stationary row of blades or secondary flow paths. To compute this interaction accurately, one must generate a computational grid for the entire 360 degree turbomachine and run a time-accurate computation at sufficient temporal resolution to capture the time-varying interaction, which can become highly expensive in terms of required CPU time. In some instances, a simplification can be made using recently developed techniques to intelligently model the unsteady interaction with only one blade passage grid. As Mach number and pressure ratio of a given compressor design increase, the requirements of time accurate simulation become increasingly demanding. In general, it becomes necessary to model half of the annulus or the full annulus in some cases. A mesh representing the entire 360 degree stage or multistage machine would likely require a resolution of ultimately billions of grid cells, if one were to de- 


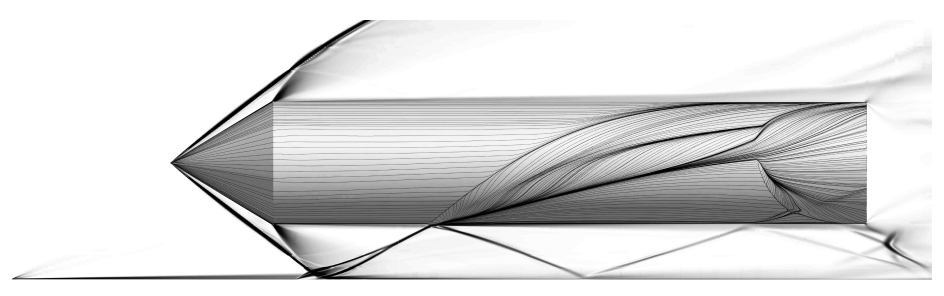

(a)

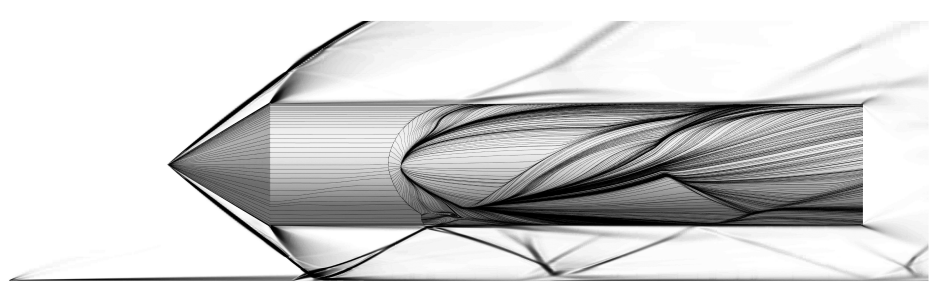

(b)

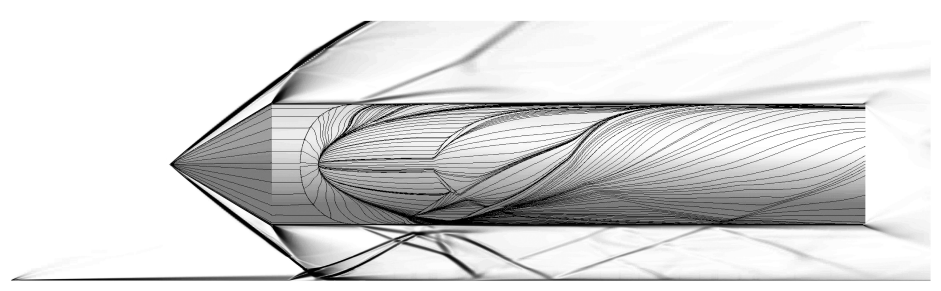

(c)

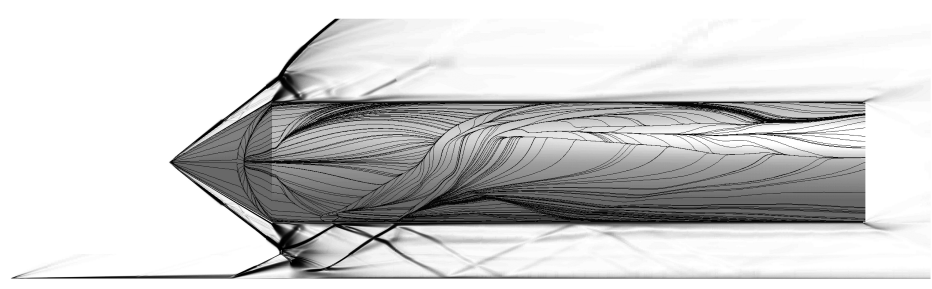

(d)

Figure 23 Predicted development of separation on inside body surface for $\theta=60^{\circ}$ for decreasing distance between bodies: $(a) \Delta z / D=3.0 ;(b) 1.8 ;$ (c) 1.4 ; and $(d) \Delta z / D$ $=1.06$ 
sire high resolution capture of the SWBLI phenomena - and higher if one were to attempt using hybrid LES approaches (coarser grids could be used, but there would be a risk of numerically dissipating turbulence to an unacceptable degree). The nonlinear harmonic (NLH) technique $[25,26]$ was developed to obtain the deterministic stress terms for a multistage environment in an efficient and accurate way. The researchers adopted a frequency-domain approach, where the Navier-Stokes equations are split into averaged and time-varying terms then time averaged, and a model is derived for resultant deterministic stress terms which depend on solution of unsteady disturbances. The procedure can be considered analogous to the method employed for deriving RANS turbulence closures. An unsteady disturbance is considered here as consisting of a number of harmonic components, each of which has a given frequency. The NLH method produces a set of quasi-linear equations to solve for all harmonics, such that an unsteady solution can be extracted from one steady solution plus additional conservation equations for the real and imaginary components of each harmonic (each member of the Fourier series). Numeca has further developed an NLH approach for practical use in 3D radial and axial turbomachine computations [27].

For the last study presented herein, RANS simulations were run for the Stage 67 endwall recirculation configuration experimentally tested at NASA Glenn Research Center in 2004. Unsteady flowfield prediction has been performed employing the NLH approach. Tip injection such as that described here involves recirculating flow driven by the pressure difference from downstream blade rows in the turbomachine (possessing higher total pressure downstream of a rotor), in order to inject a high-speed jet of fluid parallel to the shroud or casing endwall just upstream of the rotor blade. Prior fundamental wall jet, SWBLI control studies $[28,29]$ have found resistance of a boundary layer to separate in the presence of a shock wave correlates with incompressible shape factor of the incoming boundary layer and local total pressure - both of which can be manipulated using wall jets. Strazisar et al. [30] demonstrated a series of axial compressor experiments where wall jet injectors were installed on the casing just upstream of the rotor leading edge (LE), concluding: "Recirculation within a highly-loaded multistage compressor provides an effective means of increasing stability across a range of operating speeds."

\subsection{Flow Conditions and Computational Grid}

The configuration studied is shown in Fig. 3 schematically, and Fig. 24 displays the computational grid. The first blade row is a transonic fan rotor, followed by a row of stators, and then a recirculation path at the shroud, aft of the stators.

Table 3 lists characteristics of the transonic compressor test case: the number of passages in each row of the stage, the rotor tip radius at LE, the ratio of hub 


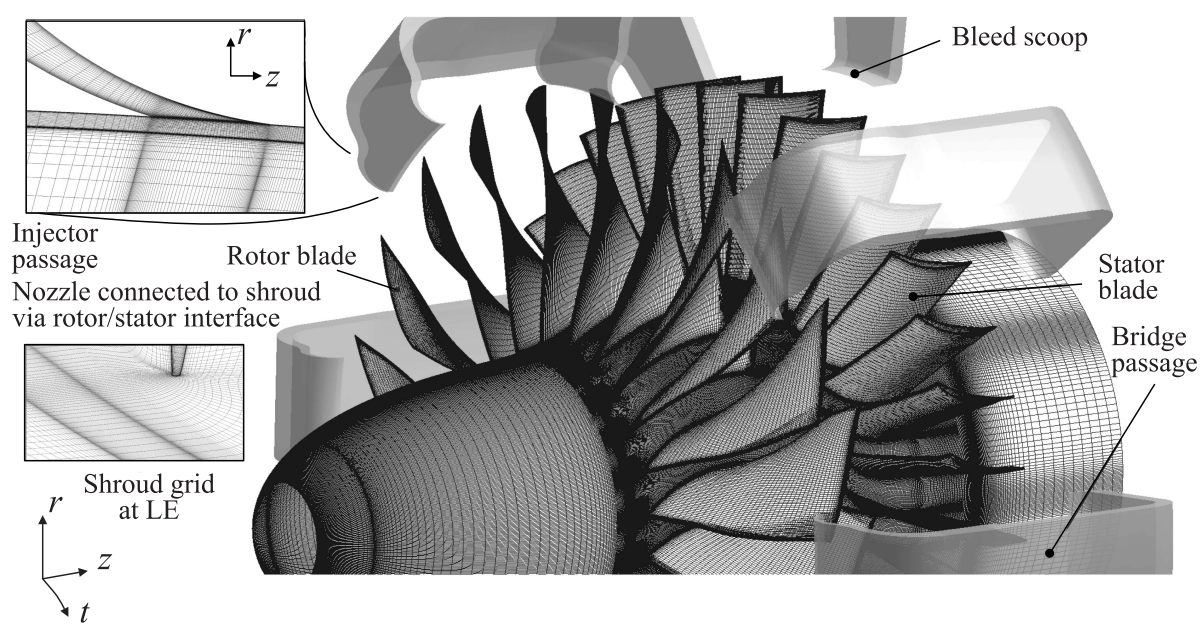

Figure 24 Transonic fan with tip injection, overview of computational domain

Table 3 Transonic fan stage geometry and operating conditions

\begin{tabular}{ccccccc}
\hline Pasages & $\begin{array}{c}r_{\text {tip }} \\
\text { at LE, cm }\end{array}$ & $\begin{array}{c}\text { Hup-tip } \\
\text { ratio }\end{array}$ & $\begin{array}{c}\text { Tip } \\
\text { clearance, mm }\end{array}$ & $\begin{array}{c}\text { Corrected } \\
\text { speed, rpm }\end{array}$ & $\operatorname{Re}_{\theta}$ & $\begin{array}{c}\text { Tip } \\
\mathrm{M}_{\text {rel }}\end{array}$ \\
\hline 22-rosign
\end{tabular}

to tip of the rotor blade, the size of clearance between the rotor tip and the casing, the design point corrected speed of the rotor in revolutions per minute (rpm), the momentum thickness Reynolds number entering the rotor, and the inflow relative Mach number at the rotor tip.

The stage configuration is designed to provide some control of the strong SWBLI effects mentioned above, by redirecting the flow using the scoop and bridge shown and injecting it as a transonic jet parallel to the casing right into the area that predominantly deteriorates first before stall, due to interaction of the tip shock system and distorted flow.

\subsection{Results}

In accordance with prior validation studies of rotor-only aerodynamics [13], good prediction of the rotor aerodynamics both near-peak operating point and near- 
stall is achieved (Figs. 25 and 26). This is illustrated by comparison of predictions with experimental data $[30,31]$ for total pressure ratio $(\mathrm{PR})$ and efficiency $\left(\eta_{\text {ad }}\right)$ from choking mass flow rate $\left(m_{\text {choke }}\right)$ to the near-stall condition (see Figs. $25 a$ and $25 b$ ) as well as by good correspondence of predicted and measured Mach number contours near the rotor tip at the near-peak and near-stall conditions (see Fig. 26a) which display a set of leading edge shocks and terminating shock downstream in the blade passage for the near-peak case and a collapsed set of shocks in front of the blade leading edge in the near-stall case. As is displayed in Fig. 26b, the predicted pitch-averaged total pressure exiting the rotor taken at the near-peak and near-stall conditions is also in good agreement with experiment [31]. Also shown are isosurface views of the rotor shock structure (density gradient magnitude, see Fig. 26c) and reversed flow at a near-stall operating point at the casing (see Fig. 26d), where significant additional separation arises as tip clearance flow passes through the terminating passage shock. The state of the flow in the near-stall regime demonstrates a particular sensitivity to the interaction of the passage shock system in the tip region of the blade and the casing endwall viscous flow. The flowfields are integrated to quantify boundary layer properties such as momentum thickness and shape factor in key regions. The figures demonstrate good prediction of the rotor aerodynamics from choking to near-stall condition and the critical flow structures at the near stall condition, specifically the passage shock system, the tip clearance leakage fluid, and the manner of flow separation that subsequently exists at the casing are displayed. This is the SWBLI region that will be next demonstrated using the wall jet injection flow control.

Figure 27 illustrates the predicted interaction of the injected jet flow with the rotor endwall fluid and tip shock system. (The computational grid employed
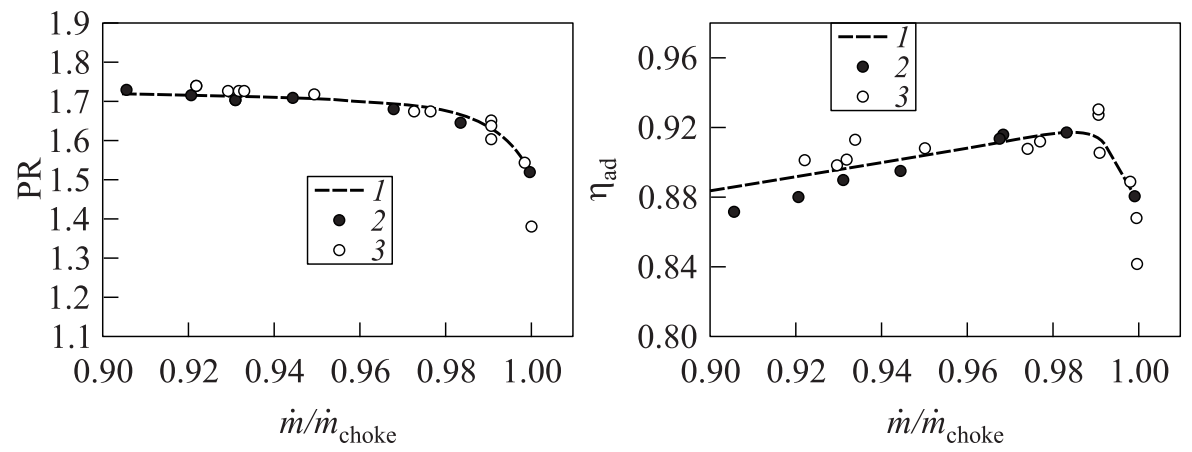

Figure 25 Rotor-only validation results: total pressure ratio $(a)$ and efficiency $(b)$ performance characteristics plotted choking mass flow rate down to near-stall regime: $1-\mathrm{CFD}\left(y_{1}^{+}=0.1-0.5 ; 9 \cdot 10^{6}\right.$ cells $) ; 2$ - experiments [30]; and $3-$ experiments [31] 


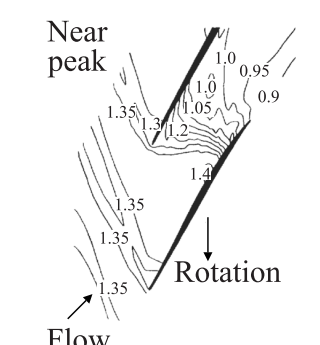

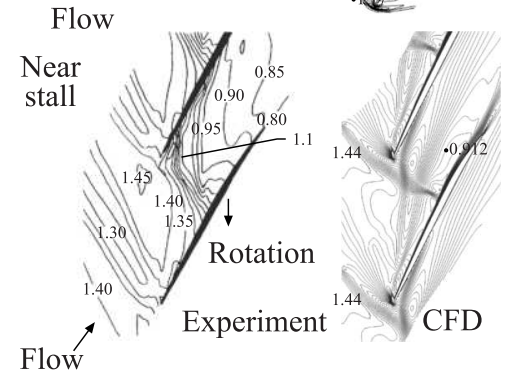

(a)

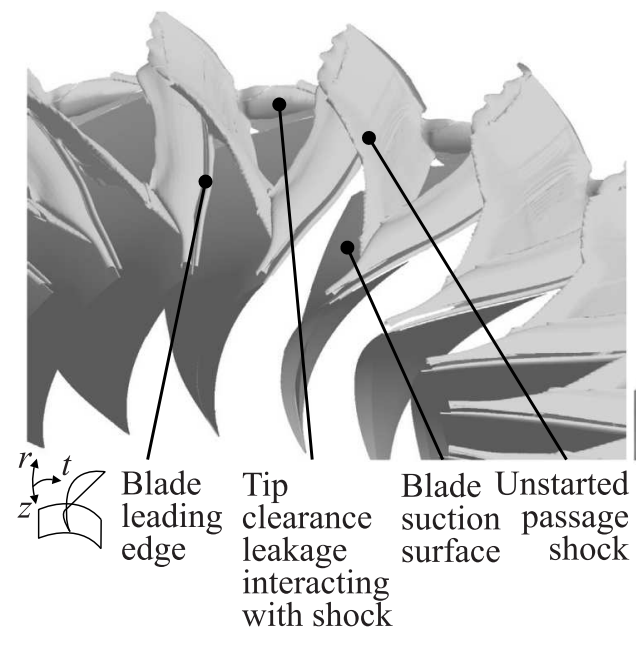

(c)

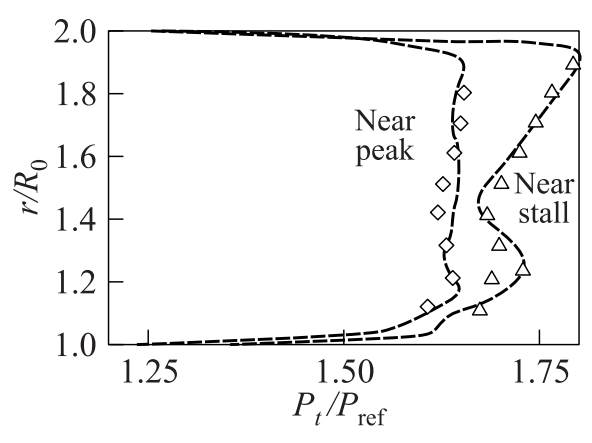

(b)

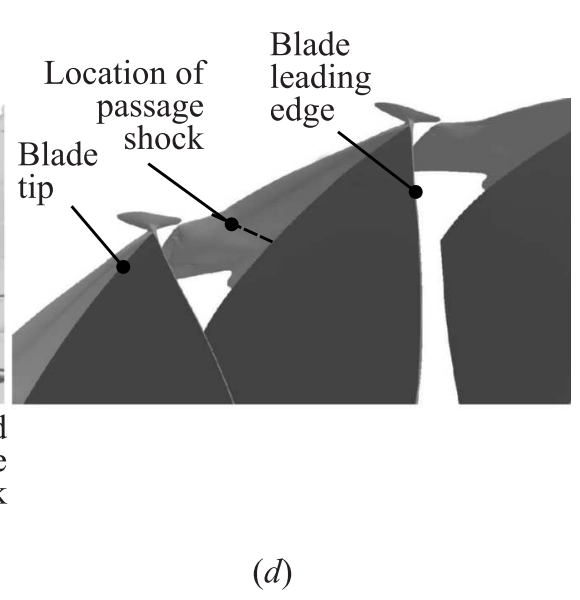

Figure 26 Rotor-only validation results: (a) tip shock system at near-peak performance and near-stall conditions - viewing radially-inward (experiments [31]); (b) total pressure profile exiting rotor (curves - CFD $\left(y_{1}^{+}=0.1-0.5 ; 9 \cdot 10^{6}\right.$ cells); symbols experiments [31]); $(c)$ isosurfaces of density gradient at near-stall operating condition showing passage shocks and tip clearance leakage vortex; and $(d)$ isosufaces of reversed flow velocity separating from casing at near-stall condition 


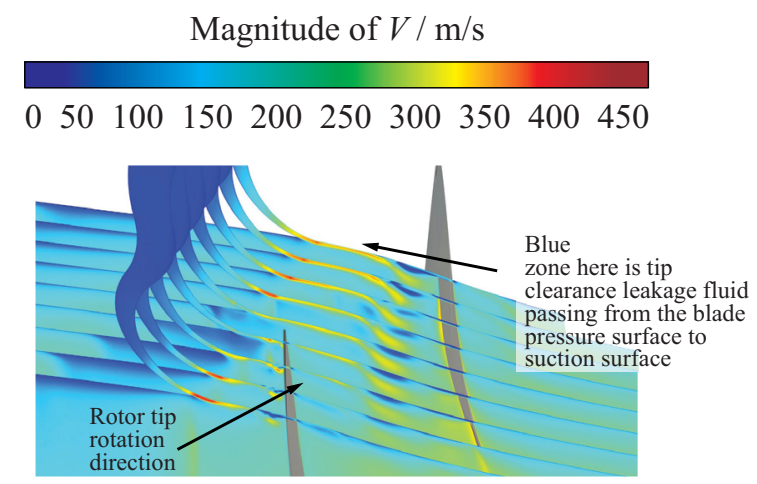

(a)

Relative Mach number
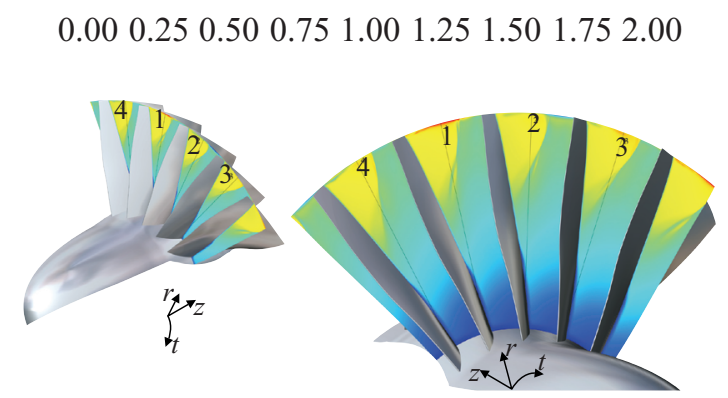

(b)

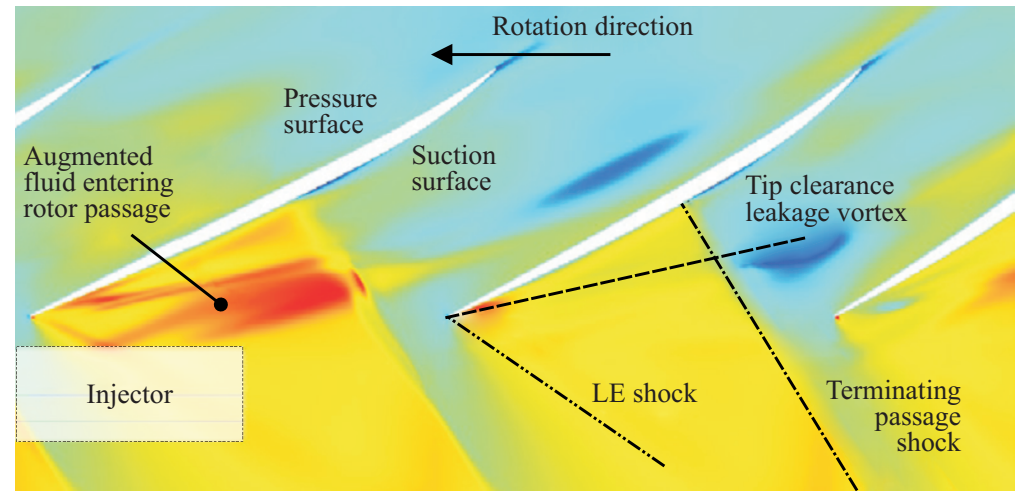

(c)

Figure 27 Predicted transonic fan tip injection details in cutting plane normal to circumferential direction $(a)$, axial cut $(b)$, and cylindrical cutting plane near rotor $\operatorname{tip}(c)$. 
for the stage configuration represented one blade passage per row and contained 80 million grid cells.) One can observe the rotor tip clearance fluid and its tendency to represent distortion and blockage to the flow entering the passage shock. Particularly, a series of circumferential cuts through the rotor blade passage and injector are displayed in Fig. $27 a$. It can be seen that the injected jet fluid augments the casing boundary layer region and then rolls up with the tip leakage vortex. Figure $27 b$ displays an axial cut through the rotor passage and plots relative Mach number contours, indicating which passages are in the path of the injectors at the given time step by the higher indicated Mach number near the blade tip.

In Fig. 27c, the near-tip region is provided using velocity magnitude plotted on a near-tip cutting plane taken parallel to the shroud or casing (in cylindrical space). The rotor tip shock system indicated in Fig. $3 b$ is shown here in terms of CFD results, where the LE and passage terminating shock wave are shown for two rotor passages. One passage is in the path of an injector, and the other is not. It can be clearly seen that as the rotor passage rotates past the injector, the local passage flow is augmented by the injected fluid and becomes more stable, whereas outside of the influence of the injector, the passage flow is distorted, and the tip leakage flow (that emanates from the pressure surface of the blade and is driven over the blade tip to the suction surface) bursts into a significantly separated zone (the dark blue zone) after passing through the terminating shock wave.

Figure $28 a$ presents the application of incipient separation criteria, where integrated casing endwall flow and rotor tip shock system strengths are quantified, in terms of momentum thickness Reynolds number for the endwall boundary layer and inviscid shock pressure ratio $\xi_{i}$ (based on normal component of Mach number entering the shock). Boundary layer profiles $1,2,3$, and 4 are identified by lines from the $3 \mathrm{D}$ views in Fig. $27 b$. The profiles are then displayed in terms of normalized velocity profiles (relative velocity in the blade chordwise direction $v_{\text {rel-chord }}$ by design speed at the blade tip $\left(U_{\text {tip-design }}\right)$ plotted vs. normalized radius $(r)$ by rotor tip radius at leading edge $\left(R_{\text {tip-LE }}\right)$ (see Fig. $\left.28 b\right)$. The profiles have then been integrated such that the boundary layer quantities (specifically, momentum thickness here) could be used for further analysis using criteria for shock-induced separation. These methods discussed in $[32,33]$ were originally developed for different 2D and 3D SWTBLI test configurations. Critical strength of the normal shock wave in conditions of $2 \mathrm{D}$ and $3 \mathrm{D}$ interaction with turbulent boundary layer was proposed to be predicted on a basis of the classic Free Interaction Theory of Chapman, Kuehn, and Larson [34]. As was shown in $[32,33]$, the critical normal shock wave strength (PR across the shock) corresponds to the plateau pressure level predicted by this theory.

Application of the method here appears to demonstrate a consistent trend for the rotating machine where the endwall flow enters the incipient separation zone in the near-stall regime (points 2 and 4 on the chart, see Fig. $28 a$ ) but wall 


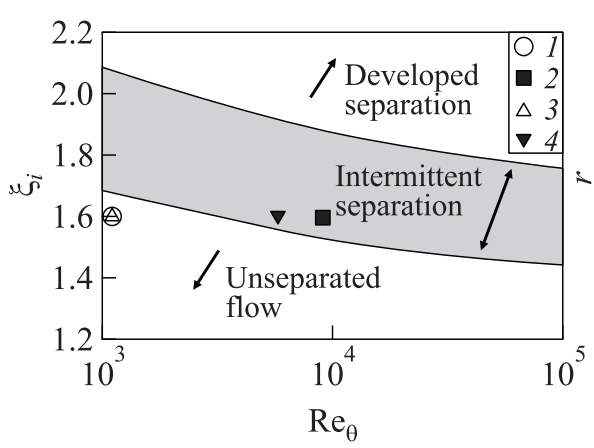

(a)

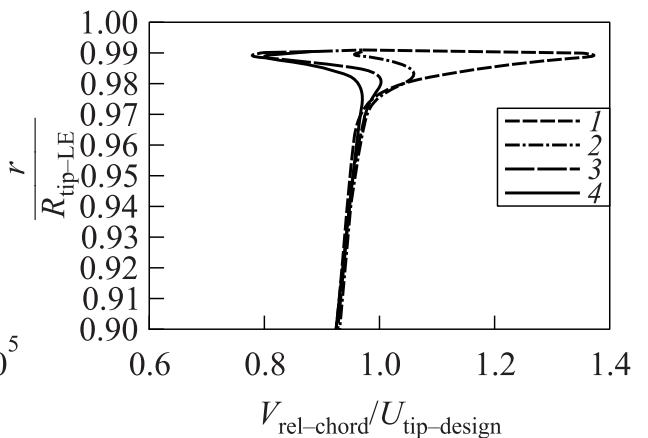

(b)

Figure 28 Application of incipient separation criteria for rotor tip shock system strength $(a)$ and the endwall flow velocity profiles $(b)$ in the near-stall regime without wall jet injection $(2,4)$ and with wall jet injection flow control $(1,3)$

jet injection flow control tends to drive the flow away from the incipient separation condition (leftward to points 1 and 3 on the chart). This is of particular interest when evaluating blade redesign, and the application of boundary layer flow control devices. Note how the injected state of the boundary layer drives the calculated momentum thickness to very low values. Although the application and scope of study was somewhat limited as presented herein, the result appears to suggest that more detailed analysis should be devoted to the application of such incipient separation criteria to rotating machinery. Such application of supersonic flow separation criteria to turbomachinery configurations has not previously been demonstrated in the open literature.

\section{CONCLUDING REMARKS}

A set of RANS computations employing the JST scheme with turbulence closure provided by the SA model has been carried out for complex 3D flow between two asymmetric fins, over two conically sharp cylindrical bodies mounted over a flat plate at varying interbody distances in Mach 4 flow and a transonic fan stage near stall. The employed scheme and turbulence model are quite effective in predicting the gas dynamic structure, topology of $3 \mathrm{D}$ separation, as well as bodies aerodynamic forces for these 3D SWTBLI flow. This paper focused on describing the interactions of shock waves and the subsequent physical mechanisms of $3 \mathrm{D}$ shock wave/boundary layer interaction, particularly taking a detailed look at $3 \mathrm{D}$ structures. Following studies should concentrate on the impact of laminar-turbulent transition as well as the unsteadiness of some configurations, 
and higher order turbulence closure should be tested for these applications. The transonic fan stage example illustrated a consistent trend of driving flow at the tip of the transonic rotor away from incipient separation using wall jet injection flow control. It seems that such techniques could be used to improve a turbomachinery designer's ability to achieve high performance in the presence of shock waves.

\section{ACKNOWLEDGMENTS}

Considered validation of the numerical computations and analysis of 3D SWTBLI flows in the vicinity of the DF and DB test configurations has been carried out by the authors of the paper on a basis of experimental studies performed at the Khristianovich Institute of Theoretical and Applied Mechanics SB RAS, Novosibirsk, Russia.

The numerical computations presented herein have been carried out at Ramgen Power Systems and the Oak Ridge Leadership Computing Facility. This material is based upon work supported by the Department of Energy under Award Number DEFE0000493. This report was prepared as an account of work sponsored by an agency of the United States Government. Neither the United States Government, nor any agency thereof, nor any of their employees makes any warranty, express or implied, or assumes any legal liability or responsibility for the accuracy, completeness, or usefulness of any information, apparatus, product, or process disclosed, or represents that its use would not infringe privately owned rights. Reference herein to any specific commercial product, process, or service by trade name, trademark, manufacturer, or otherwise does not necessarily constitute or imply its endorsement, recommendation, or favoring by the United States Government or any agency thereof. The views and opinions of authors expressed herein do not necessarily state or reflect those of the United States Government or any agency thereof.

This research used resources of the Oak Ridge Leadership Computing Facility at the Oak Ridge National Laboratory, which is supported by the Office of Science of the U.S. Department of Energy under Contract No. DEAC05-00OR22725.

\section{REFERENCES}

1. Dolling, D. S. 2001. Fifty years of shock-wave / boundary-layer interaction research: What next? AIAA J. 39(8):1517-31.

2. Zheltovodov, A. A., and D.D. Knight. 2011. Ideal-gas shock wave-turbulent boundary-layer interactions in supersonic flows and their modelling: Threedimensional interactions. In: Shock wave-boundary-layer interactions. Eds. 
H. Babinsky and J.K. Harvey. Cambridge aerospace ser. New York, NY: Cambridge University Press. 202-58.

3. Dolling, D. S. 1998. High-speed turbulent separated flows: Consistency of mathematical models and flow physics. AIAA J. 36(5):725-32.

4. Knight, D. D., H. Yan, A. G. Panaras, and A. A. Zheltovodov. 2003. Advances in CFD prediction of shock wave turbulent boundary layer interactions. In: Progress in aerospace sciences. Oxford: Pergamon Press. 39:121-84.

5. Zheltovodov, A. A. 2006. Some advances in research of shock wave turbulent boundary layer interactions. AIAA Paper No. 2006-0496.

6. Knight, D.D., and A.A. Zheltovodov. 2011. Ideal-gas shock wave-turbulent boundary-layer interactions (STBLIs) in supersonic flows and their modelling: Two-dimensional interactions. In: Shock wave-boundary-layer interactions. Eds. H. Babinsky and J. K. Harvey. Cambridge aerospace ser. New York, NY: Cambridge University Press. 137-201.

7. Spalart, P. R., W.-H. Jou, M. Strelets, and S. R. Allmaras. 1997. Comments on the feasibility of LES for wings, and on a hybrid RANS/LES approach. In: Advances in DNS/LES. Eds. C. Liuand and Z. Liu. Columbus OH: Greyden. Press.

8. Edwards, J. R., J-Il. Choi, and J. A. Boles. 2008. Large-eddy/Reynolds-averaged Navier-Stokes simulation of a Mach 5 compression-corner interaction. AIAA J. 46(4):977-91.

9. Loginov, M. S., N. A. Adams, and A. A. Zheltovodov. 2006. Large-eddy simulation of shock-wave / turbulent-boundary layer interaction. J. Fluid Mech. 565:135-69.

10. Hakimi, N. 1997. Preconditioning methods for time dependent Navier-Stokes equations - application to environmental and low speed flows. Ph.D. Thesis. Brussel: Department of Fluid Mechanics, Vrije Universiteit.

11. Jameson, A., W. Schmidt, and E. Turkel. 1981. Numerical solutions of the Euler equations by finite volume methods using Runge-Kutta time stepping schemes. AIAA Paper No. 81-1259.

12. Spalart, P. R., and S. R. Allmaras. 1992. A one-equation turbulence model for aerodynamic flows. AIAA Paper No. 92-0439.

13. Grosvenor, A.D. 2007. RANS prediction of transonic compressive rotor performance near stall. ASME Paper No. GT2007-27691.

14. Grosvenor, A.D. 2008. Numerical studies toward prediction, analysis and treatment of SWBLI in transonic compressors. 14th Conference (International) on the Methods of Aerophysical Research Proceedings. Section 2. Novosibirsk, Russia. CD. ISBN 978-5-98901-040-0. $10 \mathrm{p}$.

15. Grosvenor, A.D., A.A. Zheltovodov, and E. K. Derunov. 2010. Verification of shock-wave / turbulent-boundary-layer-interaction calculations in conditions of aerodynamic interference of two bodies of revolution with a flat surface. 15th Conference (International) on the Methods of Aerophysical Research Proceedings. Section 3. Novosibirsk, Russia. CD. ISBN 978-5-98901-085-1. 14 p.

16. Grosvenor, A.D., A. A. Zheltovodov, and E. K. Derunov. 2012. Numerical prediction of 3D shock-induced turbulent flow separation surrounding bodies of revolution adjacent to a flat surface. In: Progress in flight physics. Eds. Ph. Rei- 
jasse, D. Knight, M. Ivanov, and I. Lipatov. EUCASS book series on advances in aerospace sciences. 3:119-40.

17. Zheltovodov, A. A., A.I. Maksimov, A. M. Shevchenko, and D. D. Knight. 1998. Topology of three-dimensional separation under the conditions of asymmetric interaction of crossing shocks and expansion waves with turbulent boundary layer. Thermophys. Aeromech. 5(4):483-503.

18. Knight, D.D., T. J. Garrison, G.S. Settles, A. A. Zheltovodov, A. M. Maksimov, A. M. Schevchenko, and S. S. Votontsov. 1995. Asymmetric crossing-shockwave / turbulent-boundary-layer interaction. AIAA J. 33(12):2241-49.

19. Derunov, E.K., A.A. Zheltovodov, and A.I. Maksimov. 2008. Development of three-dimensional turbulent separation in the neighborhood of incident crossing shock waves. Thermophys. Aeromech. 15(1):29-53.

20. Derunov, E. K., V. F. Volkov, A. A. Zheltovodov, and A. I. Maksimov. 2009. Analysis of supersonic flow around two bodies of revolution near a surface. Thermophys. Aeromech. 16(1):13-36.

21. Schmisseur, J. D., D. V. Gaitonde, and A. A. Zheltovodov. 2000. Exploration of 3 -D shock turbulent boundary layer interactions through combined experimental/computational analysis. AIAA Paper No. 2000-2378.

22. Hah, C.H., D. C. Rabe, and A.R. Wadia. 2004 Role of tip-leakage vortices and passage shock in stall inception in a swept transonic compressor rotor. ASME Paper No. GT2004-53867.

23. Vo, H. D., C. S. Tan, and E. M. Greitzer. 2005. Criteria for spike initiated rotating stall. ASME Paper No. GT2005-68374.

24. Broichhausen, K. D., and K. U. Ziegler. 2005. Supersonic and transonic compressors: Past, status and technology trends. ASME Paper No. GT2005-69067.

25. Chen, T., P. Vasanthakumar, and L. He. 2001. Analysis of unsteady blade row interaction using nonlinear harmonic approach. J. Propul. Power 17(3):651-58.

26. He, L., T. Chen, R. G. Wells, Y.S. Li, and W. Ning. 2002. Analysis of rotorrotor and stator-stator interferences in multi-stage turbomachines. ASME Papers No. GT2002-30355.

27. Vilmin, S., E. Lorrain, Ch. Hirsch, and M. Swoboda. 2006. Unsteady flow modeling across the rotor/stator interface using the nonlinear harmonic method. ASME Paper No. GT2006-90210.

28. Wong, W.F., and G. R. Hall. 1975. Suppression of strong shock boundary layer interaction in supersonic inlets by boundary layer blowing. AIAA Paper No. 751209.

29. Tindell, R. H., and B.P. Willis. 1997. Experimental investigation of blowing for controlling oblique shock / boundary layer interactions. AIAA Paper No. 97-2642.

30. Strazisar, A. J., M. M. Bright, S. Thorp, D. E. Culley, and K. L. Suder. 2004. Compressor stall control through endwall recirculation. ASME Paper No. GT200454295.

31. Strazisar, A. J., J.R. Wood, M.D. Hathaway, and K.L. Suder. 1989. Laser anemometer measurements in a transonic axial-flow fan rotor. NASA TP-2879. 
32. Zheltovodov, A. A., A.I. Maksimov, E. Kh. Shilein, R. Dvorak, and P. Safarik. 1991. Development of two-dimensional analogies comfortably to three-dimensional separated flows. Separated Flows and Jets IUTAM Symposium. Eds. V. V. Kozlov and A. V. Dovgal. Berlin-Heidelberg: Springer-Verlag. 365-68.

33. Zheltovodov, A.A. 1996. Shock waves/turbulent boundary-layer interactions fundamental studies and applications. AIAA Paper No. 96-1977.

34. Chapman, D. R., H. K. Kuehn, and H. K. Larson. 1958. Investigation of separated flows in supersonic and subsonic streams with emphasis on the effect of transition. NACA Report No. 1356. 